Supporting Information for

\title{
Synthesis and Molecular Recognition of Pyrenophanes with Polycationic or Amphiphilic Functionalities: Artificial Plate-Shaped Cavitant Incorporating Arenes and Nucleotides in Water
}

Hajime Abe, Yosuke Mawatari, Haruna Teraoka, Kazuhisa Fujimoto, and Masahiko Inouye*

Contents

Preparation of pyrenophanes and intermediates

$$
(1 b-e, 5-13,19 b, d, e, \text { and 20b) }
$$

Preparation of monopyrenyl guests (21 and 23) 


\section{Preparation of Pyrenophanes and Intermediates (See Scheme 1 in the text)}

Methyl 3-[Bis[3-(dimethylamino)propyl]amino]propanoate. A methanol (150 $\mathrm{mL})$ solution of $\mathrm{N}, \mathrm{N}$-bis[3-(dimethylamino)propyl]amine (15 g, $80 \mathrm{mmol})$ and methyl acrylate (20 g, $234 \mathrm{mmol}$ ) was stirred at room temperature for $4 \mathrm{~h}$. After removal of the solvent by a rotary evaporator, the residue was subjected to column chromatography (activated alumina, $\left.\mathrm{CH}_{2} \mathrm{Cl}_{2}: \mathrm{MeOH}=50: 1\right)$ to afford methyl 3-[bis[3(dimethylamino)propyl]amino]propanoate as colorless oil (19 g, 88\%). ${ }^{1} \mathrm{H}$ NMR (400 $\left.\mathrm{MHz}, \mathrm{CDCl}_{3}\right) \delta 3.67(\mathrm{~s}, 3 \mathrm{H}), 2.78(\mathrm{t}, J=7.3 \mathrm{~Hz}, 2 \mathrm{H}), 2.47-2.41(\mathrm{~m}, 6 \mathrm{H}), 2.27-2.19(\mathrm{~m}$, $4 \mathrm{H}), 2.22(\mathrm{~s}, 12 \mathrm{H}), 1.64-1.54(\mathrm{~m}, 4 \mathrm{H})$; IR (KBr) $1741 \mathrm{~cm}^{-1}$; FABMS (3-nitrobenzyl alcohol) $m / z(\%) 273\left(\mathrm{M}^{+}, 100\right)$.

3-[Bis[3-(dimethylamino)propyl]amino]-1-propanol. To a THF $(270 \mathrm{~mL})$ suspension of $\mathrm{LiAlH}_{4}(12.5 \mathrm{~g}, 330 \mathrm{mmol})$ was added a THF $(60 \mathrm{~mL})$ solution of methyl 3-[bis[3-(dimethylamino)propyl]amino]propanoate $(19 \mathrm{~g}, 70 \mathrm{mmol})$ dropwise at $0{ }^{\circ} \mathrm{C}$. Then, the reaction mixture was refluxed for $17 \mathrm{~h}$ and cooled again to $0{ }^{\circ} \mathrm{C}$. Aqueous $10 \%$ $\mathrm{NaOH}$ was carefully added dropwise until no more hydrogen evolved, and the mixture was additionally stirred at room temperature for $2 \mathrm{~h}$. The resulted suspension was filtered, and after concentration the filtrate was acidified to $\mathrm{pH} 1$ with aqueous $1 \mathrm{~N} \mathrm{HCl}$ and washed with $\mathrm{CH}_{2} \mathrm{Cl}_{2}$, then basified to $\mathrm{pH} 11$ with aqueous $10 \% \mathrm{NaOH}$ and extracted with $\mathrm{CH}_{2} \mathrm{Cl}_{2}$. The $\mathrm{CH}_{2} \mathrm{Cl}_{2}$ extract was evaporated to give 3-[bis[3-(dimethylamino)propyl]amino]-1-propanol as colorless oil (11 g, 65\%). $\quad{ }^{1} \mathrm{H}$ NMR (400 MHz, $\left.\mathrm{CDCl}_{3}\right) \delta 3.78(\mathrm{t}, J=5.5 \mathrm{~Hz}, 2 \mathrm{H}), 2.64$ (t, $J=5.5 \mathrm{~Hz}, 2 \mathrm{H}), 2.47(\mathrm{t}, J=7.3 \mathrm{~Hz}, 4 \mathrm{H}), 2.27(\mathrm{t}, J=7.3 \mathrm{~Hz}, 4 \mathrm{H}), 2.24(\mathrm{~s}, 12 \mathrm{H})$, 1.70-1.62 (m, $6 \mathrm{H})$; FABMS (3-nitrobenzyl alcohol) $m / z(\%) 246\left(\mathrm{M}+\mathrm{H}^{+}, 100\right)$.

\section{1-[3-[Bis[3-(dimethylamino)propyl]amino]propyloxy]-3,5-bis(2-propynyloxymethyl)} benzene (5). To a THF (20 mL) solution of 3-[bis[3-(dimethylamino)propyl]amino]1-propanol (0.91 g, $3.7 \mathrm{mmol}), 4^{1}(1.7 \mathrm{~g}, 7.4 \mathrm{mmol})$, and $\mathrm{Ph}_{3} \mathrm{P}(0.97 \mathrm{~g}, 3.7 \mathrm{mmol})$ was added diethyl azodicarboxylate $(0.64 \mathrm{~g}, 3.7 \mathrm{mmol})$ dropwise at $0{ }^{\circ} \mathrm{C}$. The reaction mixture was stirred at room temperature for $20 \mathrm{~h}$ and concentrated. The residue was acidified to $\mathrm{pH} 1$ with aqueous $1 \mathrm{~N} \mathrm{HCl}$ and washed with $\mathrm{CH}_{2} \mathrm{Cl}_{2}$, then basified to $\mathrm{pH} 11$ with an aqueous $10 \% \mathrm{NaOH}$ and extracted with $\mathrm{CH}_{2} \mathrm{Cl}_{2}$. The $\mathrm{CH}_{2} \mathrm{Cl}_{2}$ extract was evaporated and subjected to column chromatography (activated alumina; $\mathrm{CH}_{2} \mathrm{Cl}_{2}$ :hexane = 20:1) to give 5 as colorless oil (1.2 g, 70\%). ${ }^{1} \mathrm{H} \mathrm{NMR}\left(400 \mathrm{MHz}, \mathrm{CDCl}_{3}\right) \delta 6.91,(\mathrm{~s}, 1 \mathrm{H})$, 6.84 (s, $2 \mathrm{H}), 4.57$ (s, $4 \mathrm{H}), 4.00(\mathrm{~d}, J=2.4 \mathrm{~Hz}, 4 \mathrm{H}), 2.60$ (t, $J=6.4 \mathrm{~Hz}, 2 \mathrm{H}), 2.48-2.43$ 
(m, $2 \mathrm{H}), 2.25-2.18$ (m, $6 \mathrm{H}), 2.21(\mathrm{~s}, 12 \mathrm{H}), 1.93-1.87$ (m, $2 \mathrm{H}), 1.63-1.58$ (m, $4 \mathrm{H})$; IR (neat) $3292,2113 \mathrm{~cm}^{-1}$; FABMS (3-nitrobenzyl alcohol) $\mathrm{m} / z(\%) 458\left(\mathrm{M}+\mathrm{H}^{+}, 100\right)$.

1-[3-[Bis[3-(dimethylamino)propyl]amino]propyloxy]-3,5-bis[3-(tributylstannyl)-2 -propynyloxymethyl]benzene (6). To a THF ( $8 \mathrm{~mL})$ solution of $5(242 \mathrm{mg}, 0.53 \mathrm{mmol})$ was added a hexane solution of $n$-BuLi $(1.07 \mathrm{mmol})$ dropwise at $0{ }^{\circ} \mathrm{C}$. After stirring at that temperature for $1 \mathrm{~h}$, to the solution was added $n-\mathrm{Bu}_{3} \mathrm{SnCl}(348 \mathrm{mg}, 1.07 \mathrm{mmol}$ ) dropwise. The reaction mixture was stirred additionally for $12 \mathrm{~h}$, being allowed to reach room temperature. The resulted mixture was concentrated, and the residue was treated with saturated aqueous $\mathrm{KF}$ and extracted with $\mathrm{Et}_{2} \mathrm{O}$. The $\mathrm{Et}_{2} \mathrm{O}$ extract was filtered and evaporated to give crude 6 as colorless oil (543 mg, 99\%), which was used in the next reaction without further purification. ${ }^{1} \mathrm{H} \mathrm{NMR}\left(400 \mathrm{MHz}, \mathrm{CDCl}_{3}\right) \delta 6.90(\mathrm{~s}, 1 \mathrm{H}), 6.83$ (s, $2 \mathrm{H}), 4.57$ (s, $4 \mathrm{H}), 4.19$ (t, $J=4.3 \mathrm{~Hz}, 2 \mathrm{H}), 3.99$ (t, $J=6.1 \mathrm{~Hz}, 2 \mathrm{H}), 2.59$ (t, $J=7.0 \mathrm{~Hz}, 2$ H), $2.46(\mathrm{t}, J=7.3 \mathrm{~Hz}, 4 \mathrm{H}), 2.30-2.09(\mathrm{~m}, 16 \mathrm{H}), 1.89$ (t, $J=7.0 \mathrm{~Hz}, 2 \mathrm{H}), 1.60-1.53$ (m, $12 \mathrm{H}), 1.37-1.30(\mathrm{~m}, 12 \mathrm{H}), 1.03-0.99(\mathrm{~m}, 12 \mathrm{H}), 0.94-0.89(\mathrm{~m}, 18 \mathrm{H})$; FABMS (3-nitrobenzyl alcohol) $m / z(\%) 1035\left(\mathrm{M}+\mathrm{H}^{+}, 100\right)$.

1,6-Diiodopyrene. This compound was prepared by a modification of the published procedure by Suzuki and Inouye et al. $^{2}$ A DMF $(200 \mathrm{~mL})$ suspension of 1,6-dibromopyrene ${ }^{3}$ (6.4 g, $\left.17.8 \mathrm{mmol}\right), \mathrm{CuI}$ (59 g, $\left.310 \mathrm{mmol}\right)$, and KI (99 g, $\left.600 \mathrm{mmol}\right)$ was stirred at $150{ }^{\circ} \mathrm{C}$ for $24 \mathrm{~h}$. After cooling to room temperature, the reaction mixture was filtered, and the precipitate was extracted with $\mathrm{CHCl}_{3}$ by a Soxhlet extractor for $30 \mathrm{~h}$. The $\mathrm{CHCl}_{3}$ extract was evaporated, and the residue was recrystallized from toluene to afford 1,6-diiodopyrene as colorless solid $(6.1 \mathrm{~g}, 75 \%)$. The characteristics of the solid were identical to those reported by Chaikovskii et al. ${ }^{4}$

1-[3-[Bis[3-(dimethylamino)propyl]amino]propyloxy]-3,5-bis[3-(6-iodopyren-1-yl)-2 -propynyloxymethyl]benzene (7). A morpholine (15 mL) suspension of 5 (100 mg, 0.22 mmol), 1,6-diiodopyrene (390 mg, $0.86 \mathrm{mmol}), \mathrm{Pd}\left(\mathrm{PPh}_{3}\right)_{4}(23 \mathrm{mg}, 0.02 \mathrm{mmol})$, and $\mathrm{CuI}$ $\left(2.2 \mathrm{mg}, 0.01 \mathrm{mmol}\right.$ ) was stirred at $110^{\circ} \mathrm{C}$ for $5 \mathrm{~h}$. The mixture was concentrated, and the resulted residue was diluted with brine and extracted with $\mathrm{CHCl}_{3}$. The $\mathrm{CHCl}_{3}$ extract was evaporated and subjected to column chromatography (silica gel; $\mathrm{CH}_{2} \mathrm{Cl}_{2}: \mathrm{MeOH}: \mathrm{Et}_{3} \mathrm{~N}=$ $30: 1: 1)$ to give 7 as viscous oil $(66 \mathrm{mg}, 27 \%) .{ }^{1} \mathrm{H} \mathrm{NMR}\left(400 \mathrm{MHz}, \mathrm{CDCl}_{3}\right) \delta 8.44(\mathrm{~d}, J=$ $8.6 \mathrm{~Hz}, 2 \mathrm{H}), 8.31$ (d, J=9.2 Hz, $2 \mathrm{H}), 8.18-7.89$ (m, $12 \mathrm{H}), 7.23$ (s, $1 \mathrm{H}), 7.00$ (s, $2 \mathrm{H})$, $4.84(\mathrm{~s}, 4 \mathrm{H}), 4.64$ (s, $4 \mathrm{H}), 4.05$ (t, $J=6.5 \mathrm{~Hz}, 2 \mathrm{H}), 2.58$ (t, $J=7.3 \mathrm{~Hz}, 2 \mathrm{H}), 2.43$ (t, $J=$ $7.3 \mathrm{~Hz}, 4 \mathrm{H}), 2.23-2.15(\mathrm{~m}, 16 \mathrm{H}), 1.91$ (t, $J=7.3 \mathrm{~Hz}, 2 \mathrm{H}), 1.70-1.56$ (m, $6 \mathrm{H})$. 
Hexaamino Pyrenophane 8. To a toluene (160 mL) solution of 7 (326 mg, $0.29 \mathrm{mmol})$ and $\mathrm{Pd}\left(\mathrm{PPh}_{3}\right)_{4}(346 \mathrm{mg}, 0.03 \mathrm{mmol})$ was added a toluene $(40 \mathrm{~mL})$ solution of $6(382 \mathrm{mg}$, $0.37 \mathrm{mmol}$ ) at room temperature. The reaction mixture was stirred at $60{ }^{\circ} \mathrm{C}$ for 2 days and concentrated. The resulted residue was diluted with brine and extracted with $\mathrm{CHCl}_{3}$. The $\mathrm{CHCl}_{3}$ extract was evaporated and subjected to column chromatography (silica gel; $\mathrm{CH}_{2} \mathrm{Cl}_{2}: 40 \% \mathrm{MeNH}_{2}(\mathrm{v} / \mathrm{v})$ in $\left.\mathrm{MeOH}=40: 1\right)$ to give 8 as solid $(2 \mathrm{mg}, 0.6 \%)$. ${ }^{1} \mathrm{H} \mathrm{NMR}$ $\left(400 \mathrm{MHz}, \mathrm{CDCl}_{3}\right) \delta 8.21(\mathrm{~d}, J=9.2 \mathrm{~Hz}, 2 \mathrm{H}), 7.78(\mathrm{~d}, J=7.9 \mathrm{~Hz}, 4 \mathrm{H}), 7.60(\mathrm{~d}, J=9.2 \mathrm{~Hz}$, $4 \mathrm{H}), 7.51(\mathrm{~d}, J=7.9 \mathrm{~Hz}, 4 \mathrm{H}), 7.44$ (s, $2 \mathrm{H}), 7.04$ (s, $4 \mathrm{H}), 4.93(\mathrm{~s}, 8 \mathrm{H}), 4.63$ (s, $8 \mathrm{H}), 4.10$ (t, $J=6.2 \mathrm{~Hz}, 4 \mathrm{H}), 2.60$ (t, $J=6.4 \mathrm{~Hz}, 2 \mathrm{H}), 2.51-2.45$ (m, $2 \mathrm{H}), 2.31-2.26$ (m, $4 \mathrm{H}), 2.21$ (s, $12 \mathrm{H}), 1.98-1.93(\mathrm{~m}, 4 \mathrm{H}), 1.67-1.59(\mathrm{~m}, 8 \mathrm{H})$; FABMS (3-nitrobenzyl alcohol) $\mathrm{m} / \mathrm{z}(\%)$ $1312\left(\mathrm{M}+\mathrm{H}^{+}, 100\right)$.

Hexaammonium Pyrenophane 1c. To a $\mathrm{CH}_{2} \mathrm{Cl}_{2}(2 \mathrm{~mL})$ solution of $8(20 \mathrm{mg}, 0.015$ mmol) was added MeOTf $(123 \mathrm{mg}, 0.75 \mathrm{mmol})$ dropwise at $0{ }^{\circ} \mathrm{C}$. The resulted precipitate was filtered and washed sequentially with hexane and ether to afford $1 \mathrm{c}$ (34 $\mathrm{mg}$, quant.). ${ }^{1} \mathrm{H}$ NMR (400 MHz, CD $\left.{ }_{3} \mathrm{OD}\right) \delta(\mathrm{d}, J=8.8 \mathrm{~Hz}, 4 \mathrm{H}), 7.67$ (d, $\left.J=7.8 \mathrm{~Hz}, 4 \mathrm{H}\right), 7.55$ (s, $\left.2 \mathrm{H}\right)$, 7.48 (d, $J=7.8 \mathrm{~Hz}, 4 \mathrm{H}), 7.44$ (d, $J=9.0 \mathrm{~Hz}, 4 \mathrm{H}), 7.13$ (s, $4 \mathrm{H}), 4.94$ (s, $8 \mathrm{H}), 4.64$ (s, $8 \mathrm{H})$, 3.31 (br s, 4 H), 3.30-2.97 (m, $56 \mathrm{H}), 2.36$ (br s, $12 \mathrm{H}$ ).

1-(Triisopropylsiloxy)-3,5-bis(2-propynyloxymethyl)benzene (9). To a $\mathrm{CH}_{2} \mathrm{Cl}_{2}$ (15 $\mathrm{mL})$ solution of $\mathbf{4}^{1}(2.1 \mathrm{~g}, 9.2 \mathrm{mmol})$ and 4-(dimethylamino)pyridine $(0.22 \mathrm{~g}, 1.8 \mathrm{mmol})$ were successively added $\mathrm{Et}_{3} \mathrm{~N}(1.0 \mathrm{~g}, 10.2 \mathrm{mmol})$ and $i-\mathrm{Pr}_{3} \mathrm{SiCl}(1.8 \mathrm{~g}, 9.2 \mathrm{mmol})$ at $0{ }^{\circ} \mathrm{C}$, and the mixture was stirred at room temperature for $12 \mathrm{~h}$. After concentration, the resulted residue was diluted with water and extracted with $\mathrm{CH}_{2} \mathrm{Cl}_{2}$. The $\mathrm{CH}_{2} \mathrm{Cl}_{2}$ extract was concentrated and subjected to column chromatography (silica gel; $\mathrm{CH}_{2} \mathrm{Cl}_{2}$ :hexane = $2: 1)$ to give 9 as colorless oil (3.5 g, quant.). $\left.{ }^{1} \mathrm{H} \mathrm{NMR} \mathrm{(270} \mathrm{MHz}, \mathrm{CDCl}_{3}\right) \delta(\mathrm{s}, 1 \mathrm{H}), 6.82$ $(\mathrm{s}, 2 \mathrm{H}), 4.55(\mathrm{~s}, 4 \mathrm{H}), 4.15(\mathrm{~d}, J=2.3 \mathrm{~Hz}, 4 \mathrm{H}), 2.46(\mathrm{t}, J=2.5 \mathrm{~Hz}, 2 \mathrm{H}), 1.55-1.13(\mathrm{~m}, 3$ H), 1.11-1.05 (m, $18 \mathrm{H})$; FABMS (3-nitrobenzyl alcohol) $\mathrm{m} / z(\%): 385\left(\mathrm{M}-\mathrm{H}^{-}, 100\right)$.

1-(Triisopropylsiloxy)-3,5-bis(3-tributylstannyl-2-propynyloxymethyl)benzene (10). This compound was prepared from $9(700 \mathrm{mg}, 1.81 \mathrm{mmol})$ in a manner similar to that described for 6; colorless oil (1.74 g, quant.), which was used in the next reaction without further purification. ${ }^{1} \mathrm{H} \mathrm{NMR}\left(270 \mathrm{MHz}, \mathrm{CDCl}_{3}\right) \delta 6.90(\mathrm{~s}, 1 \mathrm{H}), 6.80(\mathrm{~s}, 2 \mathrm{H}), 4.55(\mathrm{~s}, 4$ $\mathrm{H}), 4.16(\mathrm{t}, J=4.3 \mathrm{~Hz}, 4 \mathrm{H}), 1.60-1.53(\mathrm{~m}, 12 \mathrm{H}), 1.37-1.30(\mathrm{~m}, 12 \mathrm{H}), 1.03-0.99(\mathrm{~m}, 12$ $\mathrm{H}), 0.94-0.89(\mathrm{~m}, 18 \mathrm{H})$. 


\section{1-(Triisopropylsiloxy)-3,5-bis[3-(6-iodopyren-1-yl)-2-propynyloxymethyl]benzene}

(11). This compound was prepared from $9(638 \mathrm{mg}, 1.65 \mathrm{mmol})$ and 1,6-diiodopyrene (3.0 $\mathrm{g}, 6.6 \mathrm{mmol})$ in a manner similar to that described for 7. Purification by column chromatography (silica gel; $\mathrm{CH}_{2} \mathrm{Cl}_{2}$ :hexane $=1: 1$ ) gave 11 as solid $(573 \mathrm{mg}, 34 \%)$. ${ }^{1} \mathrm{H}$ NMR (270 MHz, $\left.\mathrm{CDCl}_{3}\right) \delta 8.30(\mathrm{~d}, J=8.9 \mathrm{~Hz}, 2 \mathrm{H}), 8.25(\mathrm{~d}, J=8.2 \mathrm{~Hz}, 2 \mathrm{H}), 8.00(\mathrm{~d}, J=$ $9.2 \mathrm{~Hz}, 2 \mathrm{H}), 7.93$ (d, $J=7.9 \mathrm{~Hz}, 2 \mathrm{H}), 7.87-7.77$ (m, $6 \mathrm{H}), 7.53$ (d, $J=8.2 \mathrm{~Hz}, 2 \mathrm{H}), 6.98$ (s, $2 \mathrm{H}), 4.83(\mathrm{~s}, 4 \mathrm{H}), 4.62(\mathrm{~s}, 4 \mathrm{H}), 1.30-1.22(\mathrm{~m}, 6 \mathrm{H}), 1.11-1.01(\mathrm{~m}, 18 \mathrm{H})$.

Triisopropylsilyl Pyrenophane 12. To a toluene $(450 \mathrm{~mL})$ solution of $\mathbf{1 0}(870 \mathrm{mg}$, $0.90 \mathrm{mmol})$ and $\mathrm{Pd}\left(\mathrm{PPh}_{3}\right)_{4}(97 \mathrm{mg}, 0.084 \mathrm{mmol})$ was added a toluene $(110 \mathrm{~mL})$ solution of $11(850 \mathrm{mg}, 0.82 \mathrm{mmol})$ at room temperature. The reaction mixture was stirred at $60{ }^{\circ} \mathrm{C}$ for 2 days and concentrated. The resulted residue was diluted with brine and extracted with $\mathrm{CHCl}_{3}$. The $\mathrm{CHCl}_{3}$ extract was evaporated and subjected to column chromatography (silica gel; $\mathrm{CH}_{2} \mathrm{Cl}_{2}$ :hexane $\left.=1: 1\right)$ to afford $12(80 \mathrm{mg}, 8 \%) . \quad{ }^{1} \mathrm{H}$ NMR $\left(270 \mathrm{MHz}, \mathrm{CDCl}_{3}\right)$ $\delta 8.18(\mathrm{~d}, J=8.9 \mathrm{~Hz}, 4 \mathrm{H}), 7.76(\mathrm{~d}, J=7.9 \mathrm{~Hz}, 4 \mathrm{H}), 7.55(\mathrm{~d}, J=9.2 \mathrm{~Hz}, 4 \mathrm{H}), 7.47(\mathrm{~m}, 6$ H), $7.02(\mathrm{~s}, 4 \mathrm{H}), 4.92(\mathrm{~s}, 8 \mathrm{H}), 4.59(\mathrm{~s}, 8 \mathrm{H}), 1.56-1.25(\mathrm{~m}, 6 \mathrm{H}), 1.17-1.10(\mathrm{~m}, 36 \mathrm{H})$; FABMS (3-nitrobenzyl alcohol) $\mathrm{m} / z(\%) 1168\left(\mathrm{M}-\mathrm{H}^{-}, 100\right)$.

Phenolic Pyrenophane 13. To a THF (2 mL) solution of $12(20 \mathrm{mg}, 0.017 \mathrm{mmol})$ were added $n-\mathrm{Bu}_{4} \mathrm{NF} \cdot \mathrm{xH}_{2} \mathrm{O}(45 \mathrm{mg}$, ca. $0.15 \mathrm{mmol})$ and a few drops of water. After stirring for $2 \mathrm{~h}$ at room temperature, the mixture was concentrated. The resulted residue was extracted with $\mathrm{CH}_{2} \mathrm{Cl}_{2}$ by a Soxhlet extractor, and the $\mathrm{CH}_{2} \mathrm{Cl}_{2}$ extract was dried over $\mathrm{MgSO}_{4}$, and concentrated. The residue was dispersed into ether, and centrifugal separation of the dispersion afforded a precipitate of $\mathbf{1 3}(10 \mathrm{mg}, 67 \%) .{ }^{1} \mathrm{H}$ NMR (270 MHz, DMSO- $\left.d_{6}\right) \delta 8.06(\mathrm{~d}, J=8.9 \mathrm{~Hz}, 4 \mathrm{H}), 7.77-7.67(\mathrm{~m}, 12 \mathrm{H}), 7.16(\mathrm{~s}, 2 \mathrm{H}), 6.82(\mathrm{~s}, 4$ $\mathrm{H}), 4.77$ (s, $8 \mathrm{H}), 4.61$ (s, $8 \mathrm{H})$.

Diamino Pyrenophane 19e: General Procedure of Reductive Amination. To a $\mathrm{CH}_{2} \mathrm{Cl}_{2}(2 \mathrm{~mL})$ solution of $\mathbf{1 8}(25 \mathrm{mg}, 0.025 \mathrm{mmol})$ were successively added $\mathrm{Et}_{2} \mathrm{NH}(22 \mathrm{mg}$, $0.30 \mathrm{mmol}), 1 \mathrm{~N} \mathrm{HCl}$ in $\mathrm{Et}_{2} \mathrm{O}(0.10 \mathrm{~mL}, 0.10 \mathrm{mmol})$, finely ground molecular sieves $4 \mathrm{~A}(5$ $\mathrm{mg}$ ), and $n-\mathrm{Bu}_{4} \mathrm{NBH}_{3} \mathrm{CN}(14 \mathrm{mg}, 0.05 \mathrm{mmol})$. The mixture was stirred at room temperature for $12 \mathrm{~h}$. To the mixture was added water, and the mixture was filtered. The resulted organic phase was stood to be separated. The aqueous phase was further extracted with $\mathrm{CH}_{2} \mathrm{Cl}_{2}$, and the combined $\mathrm{CH}_{2} \mathrm{Cl}_{2}$ layer was concentrated and subjected to column chromatography (silica gel; $\left.\mathrm{CH}_{2} \mathrm{Cl}_{2}: \mathrm{Et}_{3} \mathrm{~N}=30: 1\right)$ to give $19 \mathrm{e}(17 \mathrm{mg}, 63 \%)$. M.p. $146-149{ }^{\circ} \mathrm{C} ;{ }^{1} \mathrm{H}$ NMR $\left(300 \mathrm{MHz}, \mathrm{CDCl}_{3}\right) \delta 8.20(\mathrm{~d}, J=9.0 \mathrm{~Hz}, 4 \mathrm{H}), 7.77(\mathrm{~d}, J=7.8 \mathrm{~Hz}, 4$ 
H), 7.59 (d, $J=9.0 \mathrm{~Hz}, 4 \mathrm{H}), 7.50(\mathrm{~d}, J=7.8 \mathrm{~Hz}, 4 \mathrm{H}), 7.44$ (s, $2 \mathrm{H}), 7.05$ (s, $4 \mathrm{H}), 4.94$ (s, $8 \mathrm{H}), 4.63(\mathrm{~s}, 8 \mathrm{H}), 4.08(\mathrm{t}, J=6.5 \mathrm{~Hz}, 4 \mathrm{H}), 2.59-2.50(\mathrm{~m}, 12 \mathrm{H}), 1.88-1.81(\mathrm{~m}, 4 \mathrm{H})$, $1.71-1.63(\mathrm{~m}, 4 \mathrm{H}), 1.05(\mathrm{t}, J=7.2 \mathrm{~Hz}, 12 \mathrm{H}) ;{ }^{13} \mathrm{C} \mathrm{NMR}\left(75 \mathrm{MHz}, \mathrm{CDCl}_{3}\right) \delta 160.00$, $138.85,131.55,130.41,129.49,127.55,125.44,124.72,122.97,121.54,117.07,114.29$, 90.51, 85.75, 70.96, 67.98, 57.39, 52.58, 46.82, 27.42, 23.59, 11.68; IR (KBr) $2215 \mathrm{~cm}^{-1}$; FABMS (3-nitrobenzyl alcohol) $\mathrm{m} / z(\%) 1111\left(\mathrm{M}+\mathrm{H}^{+}, 100\right)$.

Hexaamino Pyrenophane 19b. This compound was prepared from 18 (10 mg, 0.01 $\mathrm{mmol}$ ) and $N, N$-bis[3-(dimethylamino)propyl]amine $(22 \mathrm{mg}, 0.12 \mathrm{mmol}$ ) by reductive amination as described for 19e. Yield 40\% (5 mg); ${ }^{1} \mathrm{H}$ NMR $\left(300 \mathrm{MHz}, \mathrm{CDCl}_{3}\right) \delta 8.20(\mathrm{~d}$, $J=9.0 \mathrm{~Hz}, 4 \mathrm{H}), 7.77$ (d, $J=7.8 \mathrm{~Hz}, 4 \mathrm{H}), 7.59$ (d, $J=8.9 \mathrm{~Hz}, 4 \mathrm{H}), 7.50$ (d, $J=7.7 \mathrm{~Hz}, 4$ H), $7.44(\mathrm{~s}, 2 \mathrm{H}), 7.04(\mathrm{~s}, 4 \mathrm{H}), 4.94(\mathrm{~s}, 8 \mathrm{H}), 4.63(\mathrm{~s}, 8 \mathrm{H}), 4.07$ (t, J=5.7 Hz, $4 \mathrm{H}), 3.75$ (s, $6 \mathrm{H}), 3.20$ (m, $4 \mathrm{H}), 2.76$ (m, $2 \mathrm{H}), 2.49$ (m, $2 \mathrm{H}), 2.35$ (m, $2 \mathrm{H}), 2.13$ (m, $2 \mathrm{H}), 1.85$ (m, 12 H); FABMS (3-nitrobenzyl alcohol) $m / z(\%) 1223\left(\mathrm{M}+\mathrm{H}^{+}, 100\right)$.

Tetraamino Pyrenophane 19d. This compound was prepared from 18 (10 mg, 0.01 mmol) and $N, N, N$ '-trimethyl-1,2-ethylenediamine (12 $\mathrm{mg}, 0.12 \mathrm{mmol}$ ) by reductive amination as described for 19e. Yield 41\% (4.6 mg); mp 123-125 ${ }^{\circ} \mathrm{C}$; ${ }^{1} \mathrm{H}$ NMR (300 $\left.\mathrm{MHz}, \mathrm{CDCl}_{3}\right) \delta 8.20(\mathrm{~d}, J=9.0 \mathrm{~Hz}, 4 \mathrm{H}), 7.77(\mathrm{~d}, J=8.1 \mathrm{~Hz}, 4 \mathrm{H}), 7.59(\mathrm{~d}, J=9.0 \mathrm{~Hz}, 4$ H), 7.50 (d, J= $7.8 \mathrm{~Hz}, 4 \mathrm{H}), 7.44(\mathrm{~s}, 2 \mathrm{H}), 7.04$ (s, $4 \mathrm{H}), 4.94(\mathrm{~s}, 8 \mathrm{H}), 4.63$ (s, $8 \mathrm{H}), 4.08$ (t, $J=6.2 \mathrm{~Hz}, 4 \mathrm{H}), 2.56-2.47$ (m, $12 \mathrm{H}), 2.30-2.27$ (m, $18 \mathrm{H}), 1.87-1.82$ (m, $4 \mathrm{H}), 1.75-1.69$ $(\mathrm{m}, 4 \mathrm{H}) ;{ }^{13} \mathrm{C}$ NMR $\left(75 \mathrm{MHz}, \mathrm{CDCl}_{3}\right) \delta 159.95,138.88,131.57,129.51,127.56,125.46$, $124.73,123.00,121.58,117.18,114.26,90.53,85.76,70.96,67.86,57.88,57.44,57.06$, 55.22, 45.66, 42.36, 27.23, 23.69; IR (KBr) $2227 \mathrm{~cm}^{-1}$; FABMS (3-nitrobenzyl alcohol) $\mathrm{m} / \mathrm{z}(\%) 1169\left(\mathrm{M}+\mathrm{H}^{+}, 100\right)$.

Azacrown Pyrenophane 20b. This compound was prepared from 18 (25 mg, 0.025 $\mathrm{mmol})$ and monoaza-18-crown-6 (79 $\mathrm{mg}, 0.30 \mathrm{mmol})$ by reductive amination as described for 19e. Yield 57\% (22 mg); mp 75-79 ${ }^{\circ} \mathrm{C} ;{ }^{1} \mathrm{H}$ NMR $\left(300 \mathrm{MHz}, \mathrm{CDCl}_{3}\right) \delta 8.20(\mathrm{~d}, J=$ $9.0 \mathrm{~Hz}, 4 \mathrm{H}), 7.78$ (d, $J=7.9 \mathrm{~Hz}, 4 \mathrm{H}), 7.59$ (d, $J=9.1 \mathrm{~Hz}, 4 \mathrm{H}), 7.50$ (d, $J=8.0 \mathrm{~Hz}, 4 \mathrm{H})$, 7.40 (s, 2 H), 7.04 (s, 4 H), 4.94 (s, 8 H), 4.63 (s, 8 H), 4.07 (t, J=6.3 Hz, 4 H), 3.68-3.61 $(\mathrm{m}, 32 \mathrm{H}), 2.80(\mathrm{t}, J=5.9 \mathrm{~Hz}, 8 \mathrm{H}), 2.61(\mathrm{t}, J=7.3 \mathrm{~Hz}, 4 \mathrm{H}), 1.86-1.81(\mathrm{~m}, 4 \mathrm{H})$, 1.69-1.67 (m, $4 \mathrm{H}) ;{ }^{13} \mathrm{C}$ NMR $\left(75 \mathrm{MHz}, \mathrm{CDCl}_{3}\right) \delta 159.93,138.84,131.53,130.39,129.46$, 127.53, 125.43, 124.70, 122.96, 121.51, 117.05, 114.22, 90.51, 85.73, 70.94, 70.82, 70.72, 70.34, 67.87, 57.39, 53.89, 27.10; IR (KBr) $2208 \mathrm{~cm}^{-1}$; FABMS (3-nitrobenzyl alcohol) $\mathrm{m} / \mathrm{z}(\%) 1491\left(\mathrm{M}+\mathrm{H}^{+}, 100\right)$. 
Diammonium Pyrenophane 1e: General Procedure for Quarternarization. To a $\mathrm{CH}_{2} \mathrm{Cl}_{2}(2 \mathrm{~mL})$ solution of 19e $(13 \mathrm{mg}, 0.012 \mathrm{mmol})$ was added MeOTf $(20 \mathrm{mg}, 0.12$ mmol) dropwise at $0{ }^{\circ} \mathrm{C}$. The resulting precipitate was filtered and washed successively with hexane and ether to afford 1e (16 mg, 90\%). Mp 176-179 ${ }^{\circ} \mathrm{C} ;{ }^{1} \mathrm{H}$ NMR $(300 \mathrm{MHz}$, $\left.\mathrm{CD}_{3} \mathrm{OD}\right) \delta 7.92(\mathrm{~d}, J=9.0 \mathrm{~Hz}, 4 \mathrm{H}), 7.68(\mathrm{~d}, J=7.8 \mathrm{~Hz}, 4 \mathrm{H}), 7.51-7.49$ (m, $\left.6 \mathrm{H}\right), 7.44$ (d, $J=9.0 \mathrm{~Hz}, 4 \mathrm{H}$ ), 7.09 (s, $4 \mathrm{H}), 4.94$ (s, 8 H), 4.64 (s, $8 \mathrm{H}), 4.15$ (br s, $4 \mathrm{H}), 3.34-3.24$ (m, $12 \mathrm{H}), 2.72(\mathrm{~s}, 12 \mathrm{H}), 1.88($ br s, $8 \mathrm{H}), 1.26(\mathrm{t}, J=7.1 \mathrm{~Hz}, 12 \mathrm{H}) ;{ }^{13} \mathrm{C}$ NMR $(75 \mathrm{MHz}$, $\left.\mathrm{CD}_{3} \mathrm{OD}\right) \delta 160.95,140.79,132.48,131.57,130.44,128.57,126.17,125.92,123.85,122.93$, $118.31,115.17,108.29,96.68,92.05,86.46,72.21,68.01,61.17,58.44,57.47,27.05,20.10$, 7.92; IR (KBr) 2925, 2856, 1598, 1459, 1265, 1166, 1029, 846, $640 \mathrm{~cm}^{-1}$.

Hexaammonium Pyrenophane 1b. A crude precipitate was prepared from 19b (35 mg, $0.040 \mathrm{mmol}$ ) by the quarternarization as described for 1e. The efforts of purification could have not succeeded for its too high polarity. Judging from its analogy to 1c, that is, precipitation behavier during the process of quarternarization, and solubility in pure water, we believe the precipitate consists of hexaammonium structure as $\mathbf{1 b}$.

Tetraammonium Pyrenophane 1d. This compound was prepared from 19d (13 mg, $0.01 \mathrm{mmol})$ by the quarternarization as described for 1e. Yield $88 \%(17 \mathrm{mg}) ; \mathrm{mp}>$ $214{ }^{\circ} \mathrm{C}(\mathrm{dec}) ;{ }^{1} \mathrm{H}$ NMR $\left(300 \mathrm{MHz}, \mathrm{CD}_{3} \mathrm{OD}\right) \delta 7.92(\mathrm{~d}, J=8.5 \mathrm{~Hz}, 4 \mathrm{H}), 7.65(\mathrm{~d}, J=7.1 \mathrm{~Hz}$, $4 \mathrm{H}), 7.50-7.40(\mathrm{~m}, 10 \mathrm{H}), 7.09$ (s, $4 \mathrm{H}), 4.93$ (s, $8 \mathrm{H}), 4.62$ (s, $8 \mathrm{H}), 4.19-4.17$ (m, $4 \mathrm{H})$, 3.34-3.24 (m, $42 \mathrm{H}), 2.09$ (br s, $4 \mathrm{H}$ ), 1.95 (br s, $4 \mathrm{H}$ ); IR (KBr) $2209 \mathrm{~cm}^{-1}$. 


\section{Preparation of Monopyrenyl Guests}

Preparation of the Monomeric Pyrene Possessing Amphiphilic Side Chains (21 in the text)

SCHEME S1. Synthesis of 21. ${ }^{a}$

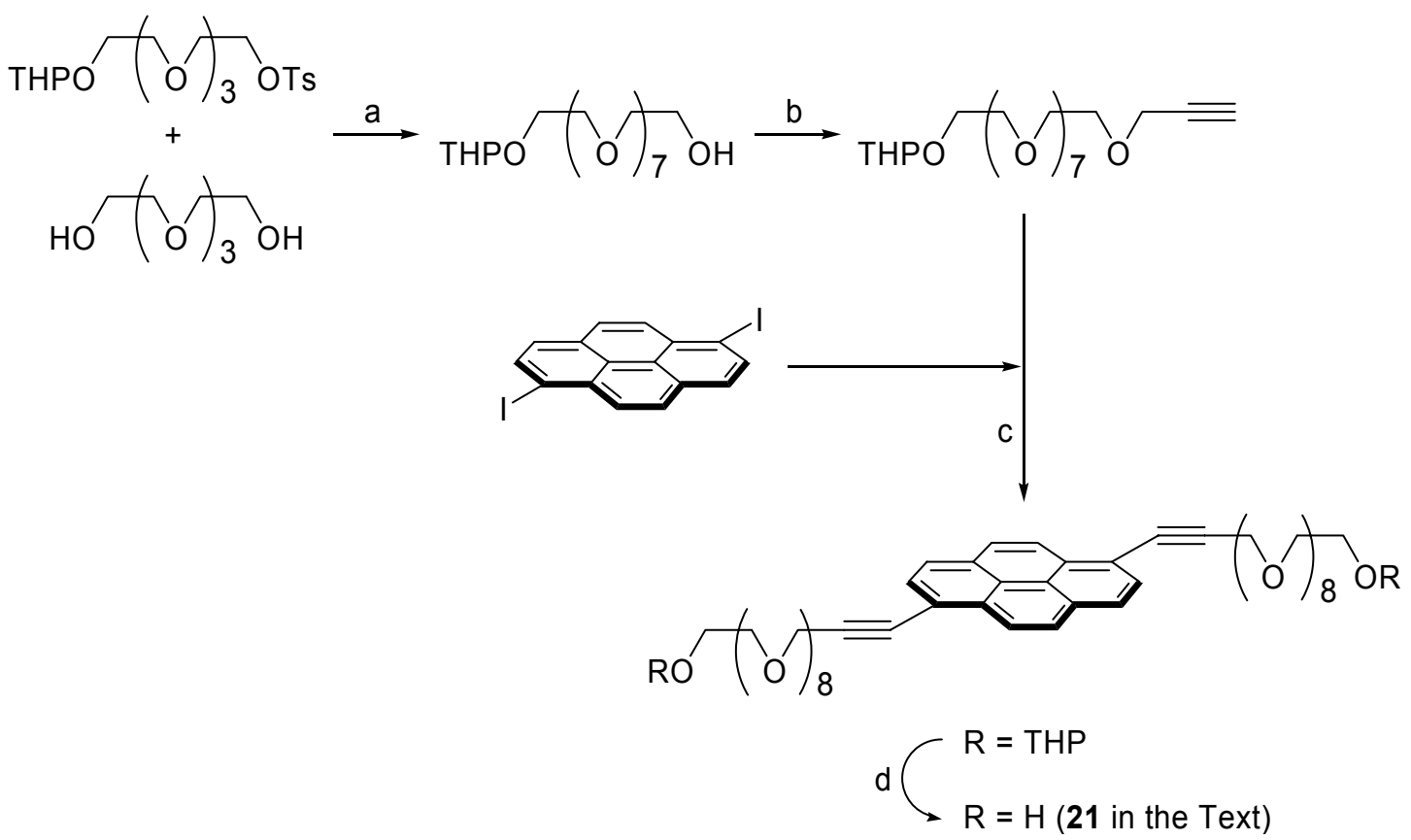

${ }^{a}$ Key: (a) NaH, THF, 2 days, 75\%; (b) NaH, propargyl bromide, THF, 1 day, 92\%; (c) 1,6-diiodopyrene, $\mathrm{PdCl}_{2}\left(\mathrm{PPh}_{3}\right)_{2}$, CuI, morpholine, $75^{\circ} \mathrm{C}, 12 \mathrm{~h}, 40 \%$; (d) $12 \mathrm{~N} \mathrm{HCl}, \mathrm{MeOH}$, r.t., $90 \%$.

23-(Tetrahydropyran-2-yloxy)-3,6,9,12,15,18,21-heptaoxatricosan-1-ol. To excess tetra(ethylene glycol) $(54.4 \mathrm{~g}, 0.28 \mathrm{~mol})$ was added $\mathrm{NaH}$ (573 mg, $0.014 \mathrm{~mol}$; commercial $60 \%$ dispersion was washed thoroughly with hexane prior to use) and stirred for $30 \mathrm{~min}$ at $0 \quad{ }^{\circ} \mathrm{C}$. To the resulted mixture was added a THF $(1 \mathrm{~mL})$ solution of 1-tosyloxy-11-(tetrahydropyran-2-yloxy)-3,6,9-trioxaundecane (3.1 g, $7.2 \mathrm{mmol}$ ) dropwise $0{ }^{\circ} \mathrm{C}$, then the mixture was gradually warmed to $50{ }^{\circ} \mathrm{C}$ and further stirred at same temperature for 2 days. After cooling, the reaction mixture was diluted with $\mathrm{CH}_{2} \mathrm{Cl}_{2}$ and 
washed with brine three times to remove the excess glycol, dried over $\mathrm{MgSO}_{4}$, and concentrated to afford 23-(tetrahydropyran-2-yloxy)-3,6,9,12,15,18,21-heptaoxadocosan1-ol as colorless oil (2.44 g, 75\%). ${ }^{1} \mathrm{HNMR}\left(300 \mathrm{MHz}, \mathrm{CDCl}_{3}\right) \delta 4.62(\mathrm{~m}, 1 \mathrm{H}), 3.89-$ 3.47 (m, $34 \mathrm{H}), 2.64$ (br s, $1 \mathrm{H}), 1.51(\mathrm{~m}, 6 \mathrm{H}) ;{ }^{13} \mathrm{C} \mathrm{NMR}\left(75 \mathrm{MHz}, \mathrm{CDCl}_{3}\right) \delta 99.06,72.69$, 70.76, 70.53, 66.83, 62.41, 61.94, 30.81, 25.68, 19.75; IR (neat) $3481 \mathrm{~cm}^{-1}$; ESI-HRMS $m / z$ calcd for $\mathrm{C}_{21} \mathrm{H}_{42} \mathrm{NaO}_{10}\left(\mathrm{M}+\mathrm{Na}^{+}\right)$: 477.2676; found: 477.2689 .

\section{7-(Tetrahydropyran-2-yloxy)-4,7,10,13,16,19,22,25-octaoxa-1-heptacosanyne.}

$\mathrm{NaH}(60 \%$ oil suspension; $212 \mathrm{mg}, 5.3 \mathrm{mmol})$ was thoroughly washed with hexane and suspended with a THF $(2.9 \mathrm{~mL})$. To the suspension was added a THF $(1.5 \mathrm{~mL})$ solution of 23-(tetrahydropyran-2-yloxy)-3,6,9,12,15,18,21-heptaoxatricosan-1-ol (2.0 g, 4.42 mmol) dropwise at $0{ }^{\circ} \mathrm{C}$. After stirring for $30 \mathrm{~min}$ at the same temperature, to the mixture propargyl bromide $(526 \mathrm{mg}, 4.42 \mathrm{mmol}$ ) was added dropwise. Being allowed to reach room temperature, the reaction mixture was additionally stirred for 1 day and concentrated. The resulted residue was dispersed in water and extracted with $\mathrm{CH}_{2} \mathrm{Cl}_{2}$. The $\mathrm{CH}_{2} \mathrm{Cl}_{2}$ extract was evaporated and subjected to column chromatography (silica gel; $\mathrm{CH}_{2} \mathrm{Cl}_{2}: \mathrm{MeOH}$ $=30: 1)$ to give 27-(tetrahydropyran-2-yloxy)-4,7,10,13,16,19,22,25-octaoxa-1heptacosanyne as colorless oil (2.0 g, 92\%). ${ }^{1} \mathrm{H} \mathrm{NMR}\left(300 \mathrm{MHz}, \mathrm{CDCl}_{3}\right) \delta 4.63(\mathrm{~m}, 1 \mathrm{H})$, $4.20(\mathrm{~d}, J=2.5 \mathrm{~Hz}, 2 \mathrm{H}), 3.89-3.83(\mathrm{~m}, 2 \mathrm{H}), 3.74-3.59(\mathrm{~m}, 40 \mathrm{H}), 3.57-3.48(\mathrm{~m}, 2 \mathrm{H})$, $2.44(\mathrm{t}, J=2.3 \mathrm{~Hz}, 1 \mathrm{H}), 1.82-1.49(\mathrm{~m}, 6 \mathrm{H}) ;{ }^{13} \mathrm{C} \mathrm{NMR}\left(75 \mathrm{MHz}, \mathrm{CDCl}_{3}\right) \delta 99.09,77.39$, 74.71, 70.78, 70.62, 69.30, 66.86, 62.44, 58.63, 30.85, 25.72, 19.78; IR (KBr) 3248, 2114 $\mathrm{cm}^{-1}$; ESI-HRMS $m / z$ calcd for $\mathrm{C}_{24} \mathrm{H}_{44} \mathrm{NaO}_{10}\left(\mathrm{M}+\mathrm{Na}^{+}\right): 515.2832$; found: 515.2797.

THP-protected Bis[octa(oxyethylene)] Monomeric Pyrene. A morpholine (3 $\mathrm{mL})$ suspention of 1,6-diiodopyrene (173 mg, $0.38 \mathrm{mmol}), \mathrm{PdCl}_{2}\left(\mathrm{PPh}_{3}\right)_{2}(27 \mathrm{mg}, 0.038 \mathrm{mmol}$ ), and $\mathrm{CuI}(3.6 \mathrm{mg}, 0.019 \mathrm{mmol})$ was stirred at $75{ }^{\circ} \mathrm{C}$ until the reaction mixture became homogeneous. To the solution was added a morpholine $(2 \mathrm{~mL})$ solution of 27-(tetrahydropyran-2-yloxy)-4,7,10,13,16,19,22,25-octaoxa-1-heptacosanyne (561 mg, $1.14 \mathrm{mmol}$ ), and the mixture was additionally stirred for $12 \mathrm{~h}$ at the same temperature. After concentration, the resulted residue was subjected to column chromatographed (silica gel; $\left.\mathrm{CH}_{2} \mathrm{Cl}_{2}: \mathrm{MeOH}=100: 1\right)$ to give THP-protected bis[octa(oxyethylene)] monomeric pyrene as oil (180 mg, 40\%). ${ }^{1} \mathrm{H}$ NMR $\left(300 \mathrm{MHz} \mathrm{CDCl}_{3}\right) \delta 8.57(\mathrm{~d}, J=9.1 \mathrm{~Hz}, 2 \mathrm{H})$, $8.12(\mathrm{~m}, 6 \mathrm{H}), 4.67(\mathrm{~s}, 4 \mathrm{H}), 4.62(\mathrm{~m}, 2 \mathrm{H}), 3.93-3.51(\mathrm{~m}, 68 \mathrm{H}), 1.80-1.52(\mathrm{~m}, 12 \mathrm{H}) ;{ }^{13} \mathrm{C}$ NMR (75 MHz, $\left.\mathrm{CDCl}_{3}\right) \delta 132.24,130.32,130.30,128.26,126.37,125.17,124.09,117.96$, $99.08,91.29,85.34,70.89,70.76,69.58,66.85,64.05,62.43,59.76,30.83,25.70,19.76$; IR 
(neat) $2220 \mathrm{~cm}^{-1}$; ESI-HRMS $\mathrm{m} / z$ calcd for $\mathrm{C}_{64} \mathrm{H}_{94} \mathrm{NaO}_{20}\left(\mathrm{M}+\mathrm{Na}^{+}\right)$: 1205.6236; found:1205.6265.

Bis[octa(oxyethylene)] Monomeric Pyrene 21. To a $\mathrm{MeOH}(17 \mathrm{~mL})$ solution of THP-protected monomeric pyrene prepared above $(822 \mathrm{mg}, 0.695 \mathrm{mmol})$ was added a several drops of conc. $\mathrm{HCl}$, and the mixture was stirred for $10 \mathrm{~min}$ at room temperature. After addition of finely ground $\mathrm{K}_{2} \mathrm{CO}_{3}$, the reaction mixture was additionally stirred for 10 min and filtered. The filterate was evaporated and subjected to reverse phase HPLC (ODS column (Shinwa Chemical Industries, Ltd.); $\mathrm{MeOH}: \mathrm{H}_{2} \mathrm{O}=5: 1$ ) to give 21 as oil (635 $\mathrm{mg}$, 90\%). $\quad{ }^{1} \mathrm{H}$ NMR (300 MHz, $\left.\mathrm{CDCl}_{3}\right) \delta 8.57(\mathrm{~d}, J=9.1 \mathrm{~Hz}, 2 \mathrm{H}), 8.12(\mathrm{~m}, 6 \mathrm{H}), 4.67(\mathrm{~s}, 4$ $\mathrm{H}), 3.92-3.90$ (m, $4 \mathrm{H}), 3.81-3.60(\mathrm{~m}, 60 \mathrm{H}) ;{ }^{13} \mathrm{CNMR}\left(75 \mathrm{MHz}, \mathrm{CDCl}_{3}\right) \delta 132.23,131.27$, $130.32,128.25,126.37,125.17,124.17,117.96,91.31,85.32,72.70,70.89,70.74,70.50$, 69.58, 61.94, 59.76; IR (neat) 3482, $2209 \mathrm{~cm}^{-1}$; ESI-HRMS $\mathrm{m} / z$ calcd for $\mathrm{C}_{54} \mathrm{H}_{78} \mathrm{NaO}_{18}(\mathrm{M}$ $+\mathrm{Na}^{+}$) 1037.5086; found: 1037.5087.

Preparation of the Monomeric Pyrene Possessing Anionic Side Chains (23 in the text)

SCHEME S2. Synthesis of 23. ${ }^{a}$

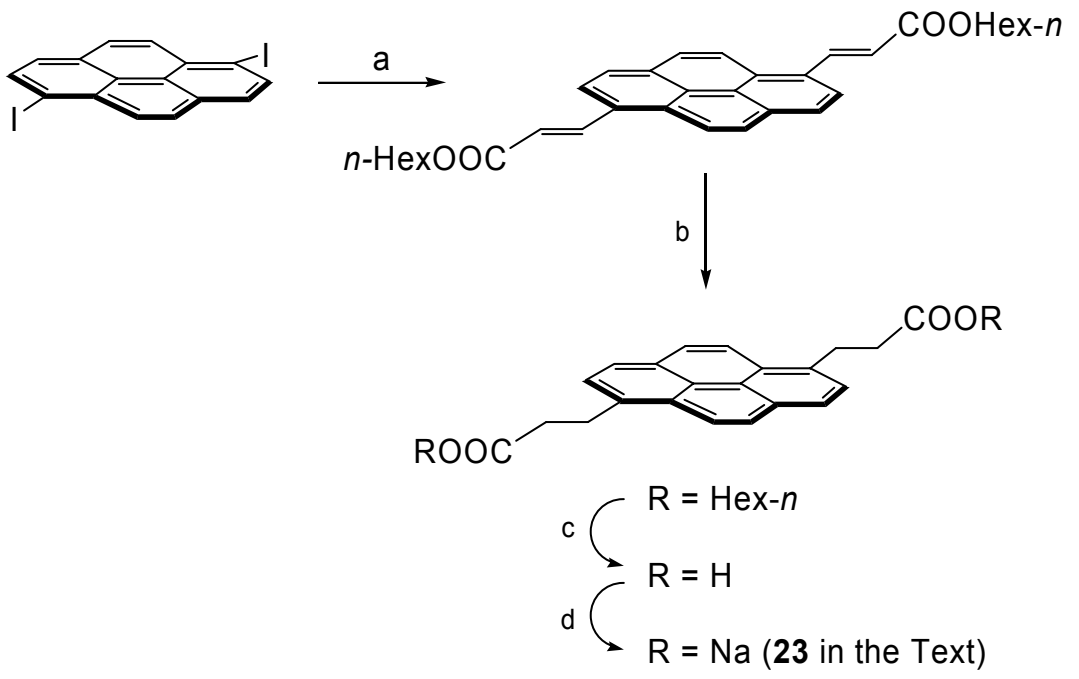

${ }^{a}$ Key: (a) acrylic acid $n$-hexyl ester, $\mathrm{Pd}(\mathrm{OAc})_{2}, \mathrm{P}(o-\mathrm{Tol})_{3}, \mathrm{Et}_{3} \mathrm{~N}, 120{ }^{\circ} \mathrm{C}, 6 \mathrm{~h}, 94 \%$; (b) $\mathrm{H}_{2}$ 40 atm, $\mathrm{PtO}_{2}$, AcOEt, 48 h, 92\%; (c) $1 \mathrm{M} \mathrm{KOH}$ in $\mathrm{MeOH}, \mathrm{H}_{2} \mathrm{O}, 80{ }^{\circ} \mathrm{C}, 20 \mathrm{~min}, 95 \%$; (d) $0.1 \mathrm{M}$ aq $\mathrm{NaOH}$, quant. 
Di-n-hexyl 1,6-Pyrenedipropenoate. $\mathrm{An} \mathrm{Et}_{3} \mathrm{~N}(5 \mathrm{~mL})$ suspension of 1,6-diiodopyrene (200 mg, $0.44 \mathrm{mmol})$, acrylic acid $n$-hexyl ester (550 mg, $3.5 \mathrm{mmol}), \mathrm{Pd}(\mathrm{OAc})_{2}(7.9 \mathrm{mg}$, $0.035 \mathrm{mmol})$, and $\mathrm{P}(o-\mathrm{Tol})_{3}(43 \mathrm{mg}, 0.14 \mathrm{mmol})$ was stirred at $120{ }^{\circ} \mathrm{C}$ for $6 \mathrm{~h}$. After concentration, the resulted residue was subjected to column chromatography (silica gel; $\mathrm{CH}_{2} \mathrm{Cl}_{2}$ :hexane $=2: 3$ ) to give di- $n$-hexyl 1,6-pyrenedipropenoate as yellow solid: (219 $\mathrm{mg}$, 94\%). $\quad \mathrm{Mp} 123-126{ }^{\circ} \mathrm{C} ;{ }^{1} \mathrm{H}$ NMR $\left(300 \mathrm{MHz}, \mathrm{CDCl}_{3}\right) \delta 8.83(\mathrm{~d}, J=15.6 \mathrm{~Hz}, 2 \mathrm{H}), 8.54$ (d, $J=9.3 \mathrm{~Hz}, 2 \mathrm{H}), 8.32(\mathrm{~d}, J=8.0 \mathrm{~Hz}, 2 \mathrm{H}), 8.23-8.16(\mathrm{~m}, 4 \mathrm{H}), 6.73(\mathrm{~d}, J=15.6 \mathrm{~Hz}, 2 \mathrm{H})$, 4.30 (t, $J=6.7 \mathrm{~Hz}, 4 \mathrm{H}), 1.80-1.76$ (m, $4 \mathrm{H}), 1.56-1.36$ (m, $12 \mathrm{H}), 0.93$ (t, $J=7.0 \mathrm{~Hz}, 6$ $\mathrm{H}) ;{ }^{13} \mathrm{CNMR}\left(75 \mathrm{MHz}, \mathrm{CDCl}_{3}\right) \delta 167.13,142.31,141.14,136.76,129.41,128.52,125.90$, 124.74, 123.68, 121.10, 109.32, 65.16, 31.78, 29.03, 26.01, 22.89, 14.36; IR (KBr) 1708, $1625 \mathrm{~cm}^{-1}$; ESI-HRMS $m / z$ calcd for $\mathrm{C}_{34} \mathrm{H}_{38} \mathrm{NaO}_{4}\left(\mathrm{M}+\mathrm{Na}^{+}\right)$533.2668; found: 533.2702.

Di-n-hexyl 1,6-Pyrenedipropanoate. In an autocrave apparatus, an AcOEt (15 mL) suspension of di- $n$-hexyl 1,6-pyrenedipropenoate $(82.0 \mathrm{mg}, 0.161 \mathrm{mmol})$ and $\mathrm{PtO}_{2}(8 \mathrm{mg}$, $0.035 \mathrm{mmol}$ ) was stirred at room temperature for $48 \mathrm{~h}$ under an $\mathrm{H}_{2}$ pressure (40 atm). The mixture was filtered through a celite bed and concentrated. The resulted residue was washed with AcOEt to give di- $n$-hexyl 1,6-pyrenedipropanoate (79 mg, 92\%). Mp 92-97 ${ }^{\circ} \mathrm{C} ;{ }^{1} \mathrm{H}$ NMR $\left(300 \mathrm{MHz}, \mathrm{CDCl}_{3}\right) \delta 8.23(\mathrm{~d}, J=9.3 \mathrm{~Hz}, 2 \mathrm{H}), 8.10(\mathrm{~m}, 4 \mathrm{H}), 7.90(\mathrm{~d}, J$ $=7.7 \mathrm{~Hz}, 2 \mathrm{H}), 4.09(\mathrm{t}, J=6.7 \mathrm{~Hz}, 4 \mathrm{H}), 3.69$ (t, $J=7.8 \mathrm{~Hz}, 4 \mathrm{H}), 2.86(\mathrm{t}, J=8.0 \mathrm{~Hz}, 4 \mathrm{H})$, $1.56(\mathrm{~m}, 4 \mathrm{H}), 1.23(\mathrm{~m}, 12 \mathrm{H}), 0.85(\mathrm{~m}, 6 \mathrm{H}) ;{ }^{13} \mathrm{C} \mathrm{NMR}\left(75 \mathrm{MHz}, \mathrm{CDCl}_{3}\right) \delta 173.05,134.69$, 129.93, 128.89, 127.76, 127.21, 125.43, 124.90, 122.44, 65.00, 31.66, 29.10, 28.82, 25.83, 22.76, 14.28; IR (KBr) 2931, 1733, $1171 \mathrm{~cm}^{-1}$; ESI-HRMS $m / z$ calcd for $\mathrm{C}_{34} \mathrm{H}_{42} \mathrm{NaO}_{4}(\mathrm{M}+$ $\mathrm{Na}^{+}$) 537.2981; found: 537.2926.

1,6-Pyrenedipropanoic Acid. Di- $n$-hexyl 1,6-pyrenedipropanoate (100 mg, 0.194 mmol) was dissolved into $1 \mathrm{M} \mathrm{KOH}$ in $\mathrm{MeOH}(4 \mathrm{~mL})$ and the solution was stirred at $80^{\circ} \mathrm{C}$ for $20 \mathrm{~min}$. The mixture was washed $\mathrm{Et}_{2} \mathrm{O}$, and then the aqueous phase was acidified with $1 \mathrm{~N} \mathrm{HCl}$ solution and extracted with a mixed solvent of AcOEt and THF. The extract was concentrated to give 1,6-pyrenedipropanoic acid (64 mg, 95\%). $\mathrm{Mp}>250{ }^{\circ} \mathrm{C} ;{ }^{1} \mathrm{H} \mathrm{NMR}$ (300 MHz, DMSO- $\left.d_{6}\right) \delta 8.30(\mathrm{~d}, J=9.6 \mathrm{~Hz}, 2 \mathrm{H}), 8.20(\mathrm{~m}, 4 \mathrm{H}), 7.97(\mathrm{~d}, J=7.8 \mathrm{~Hz}, 2 \mathrm{H})$, $3.58(\mathrm{t}, J=7.7 \mathrm{~Hz}, 4 \mathrm{H}), 2.75(\mathrm{t}, J=7.7 \mathrm{~Hz}, 4 \mathrm{H}) ;{ }^{13} \mathrm{C}$ NMR $\left(75 \mathrm{MHz}, \mathrm{DMSO}-d_{6}\right) \delta 173.47$, $134.96,129.04,128.08,127.39,127.10,124.60,124.30,122.29,35.55,28.05$; IR (KBr) 3036 (br), $1708 \mathrm{~cm}^{-1}$; ESI-HRMS $m / z$ calcd for $\mathrm{C}_{22} \mathrm{H}_{18} \mathrm{KO}_{4}\left(\mathrm{M}+\mathrm{K}^{+}\right) 385.0842$; found: 385.0815 . 
Disodium 1,6-Pyrenedipropanoate 23. 1,6-Pyrenedipropanoic acid (98 mg, 0.283 mmol) was dissolved into aqueous $0.1 \mathrm{M} \mathrm{NaOH}(5.7 \mathrm{ml}, 0.566 \mathrm{mmol})$, and the mixture was stirred at room temperature for $10 \mathrm{~min}$. Thus, the mixture was lyophilized and evapolated to give 23 (110 mg, quant.). $\quad \mathrm{Mp}>250{ }^{\circ} \mathrm{C} ;{ }^{1} \mathrm{HNMR}\left(300 \mathrm{MHz}, \mathrm{D}_{2} \mathrm{O}\right) \delta 8.25$ (d, $J=10.2$ $\mathrm{Hz}, 2 \mathrm{H}), 8.12$ (m, $4 \mathrm{H}), 7.93$ (d, $J=6.3 \mathrm{~Hz}, 2 \mathrm{H}), 3.58$ (t, $J=7.5 \mathrm{~Hz}, 4 \mathrm{H}), 2.70$ (t, $J=7.5$ $\mathrm{Hz}, 4 \mathrm{H}) ;{ }^{13} \mathrm{C}$ NMR $\left(75 \mathrm{MHz}, \mathrm{D}_{2} \mathrm{O}\right) \delta 182.22,135.75,129.02,127.97,127.21,126.87$, 124.65, 124.20, 122.19, 39.40, 29.81; IR(KBr): 1565, $1421 \mathrm{~cm}^{-1}$; ESI-HRMS $m / z$ calcd for $\mathrm{C}_{22} \mathrm{H}_{17} \mathrm{Na}_{2} \mathrm{O}_{4}\left(\mathrm{M}+\mathrm{H}^{+}\right)$391.0922; found: 391.0958 .

\section{References}

(1) Inouye, M.; Fujimoto, K.; Furusyo, M.; Nakazumi, H. J. Am. Chem. Soc. 1999, 121, $1452-1458$.

(2) Suzuki, H.; Kondo, A.; Inouye, M.; Ogawa, T. Synthesis 1986, 121-122.

(3) Grimshaw, J.; Trocha-Grimshaw, J. J. Chem. Soc., Perkin Trans. 1 1972, 1622-1623.

(4) Chaikovskii, V. K.; Novikov, A. N.; Sarycheva, T. A. Zh. Org. Khim. 1985, 21, 1947-1950. 


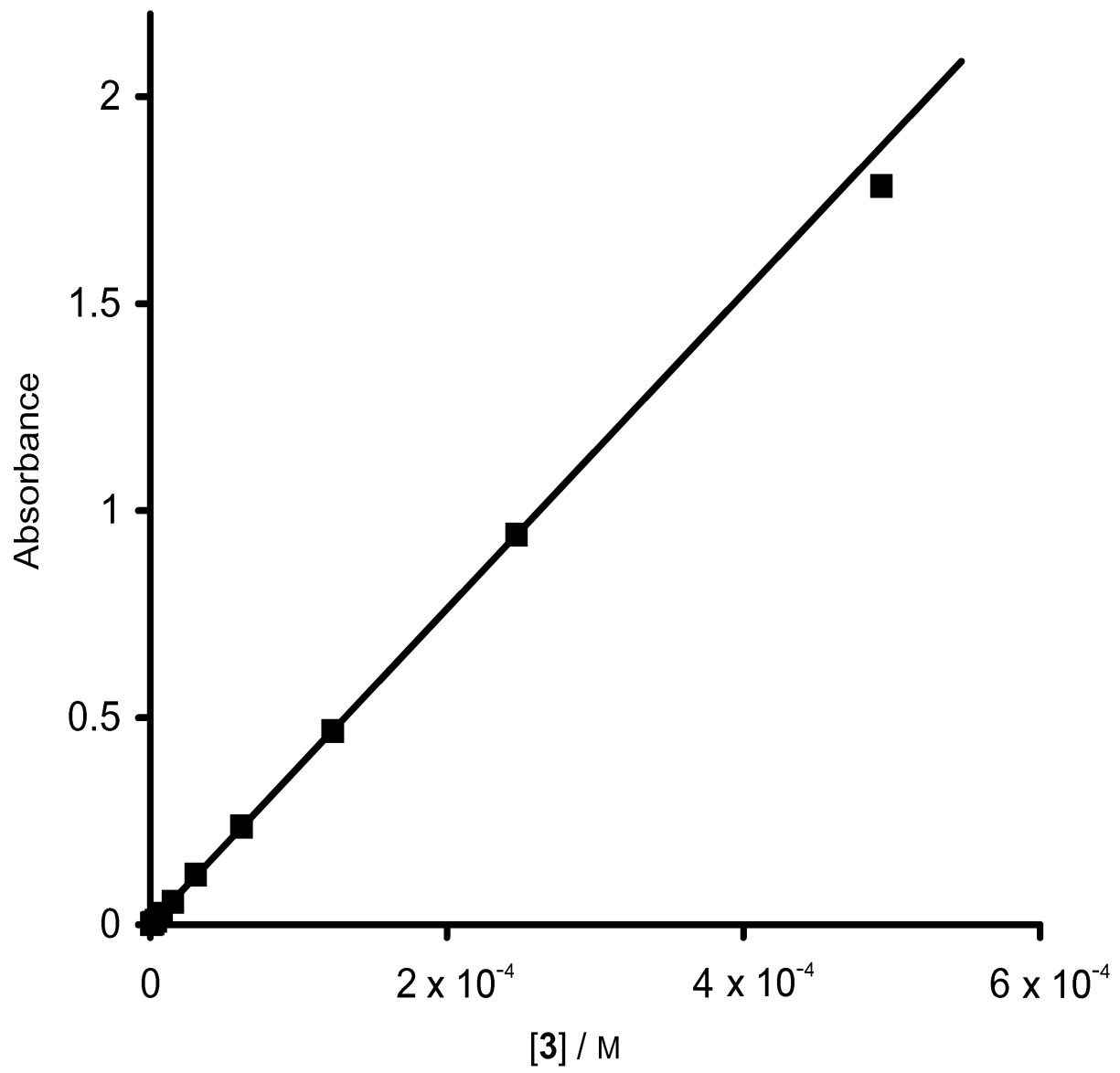

FIGURE S1. The relationship between the absorbance at $391 \mathrm{~nm}$ and the concentration of 3 in water. Light-path length was $0.1 \mathrm{~cm}\left([3]>3 \times 10^{-5} \mathrm{M}\right)$ and $1.0 \mathrm{~cm}\left([3]<3 \times 10^{-5}\right.$ M) normalized to the former. 


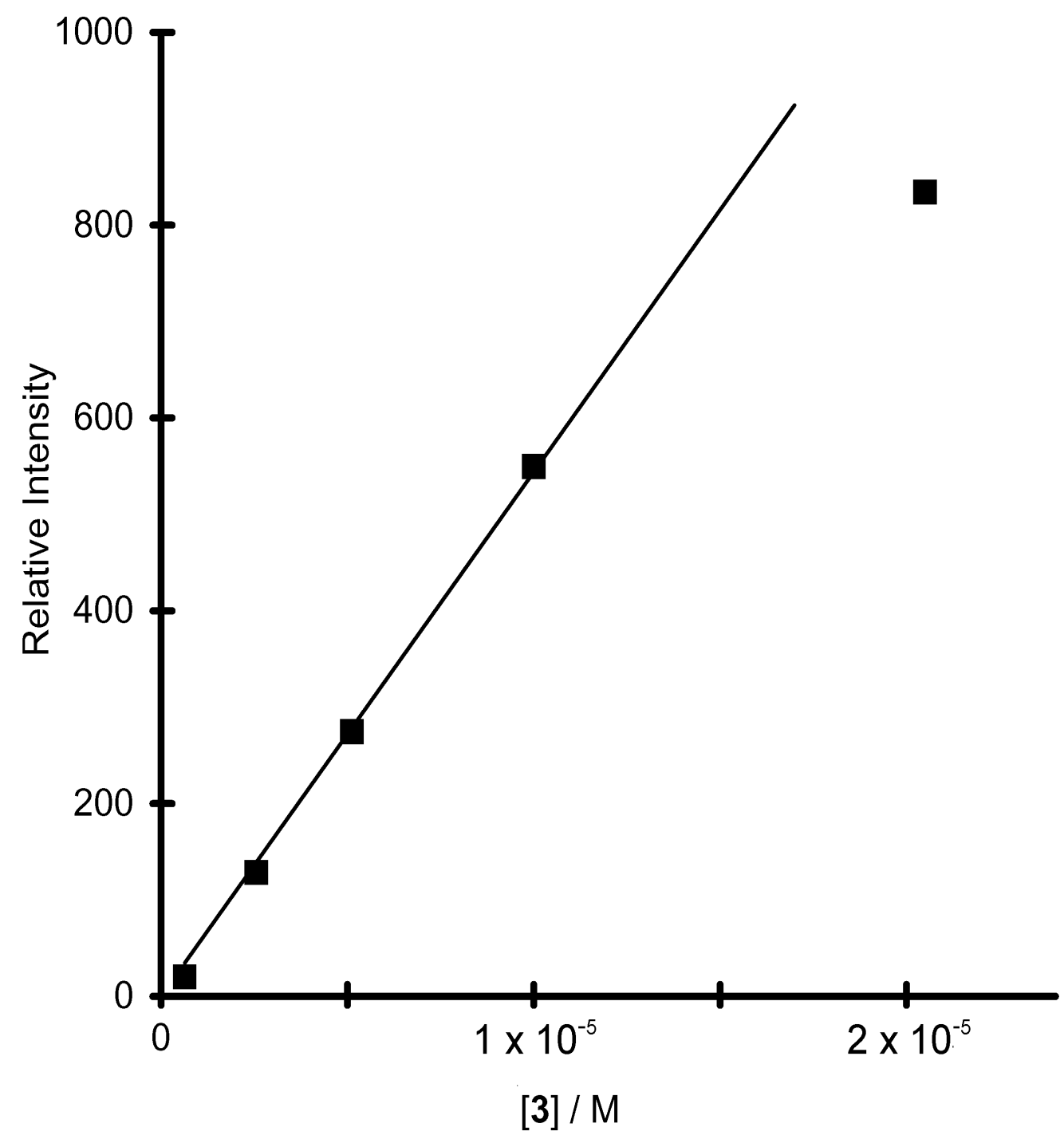

FIGURE S2. The relationship between the emission intensity at $520 \mathrm{~nm}$ and the concentration of $\mathbf{3}$ in water. The excitation wavelength was $370 \mathrm{~nm}$. 
${ }^{1}$ H NMR spectra of 1d, 1e, 2a, 3, 5-18, 19b, 19d, 19e, 20a, and 20b

Tetraammonium Pyrenophane 1d
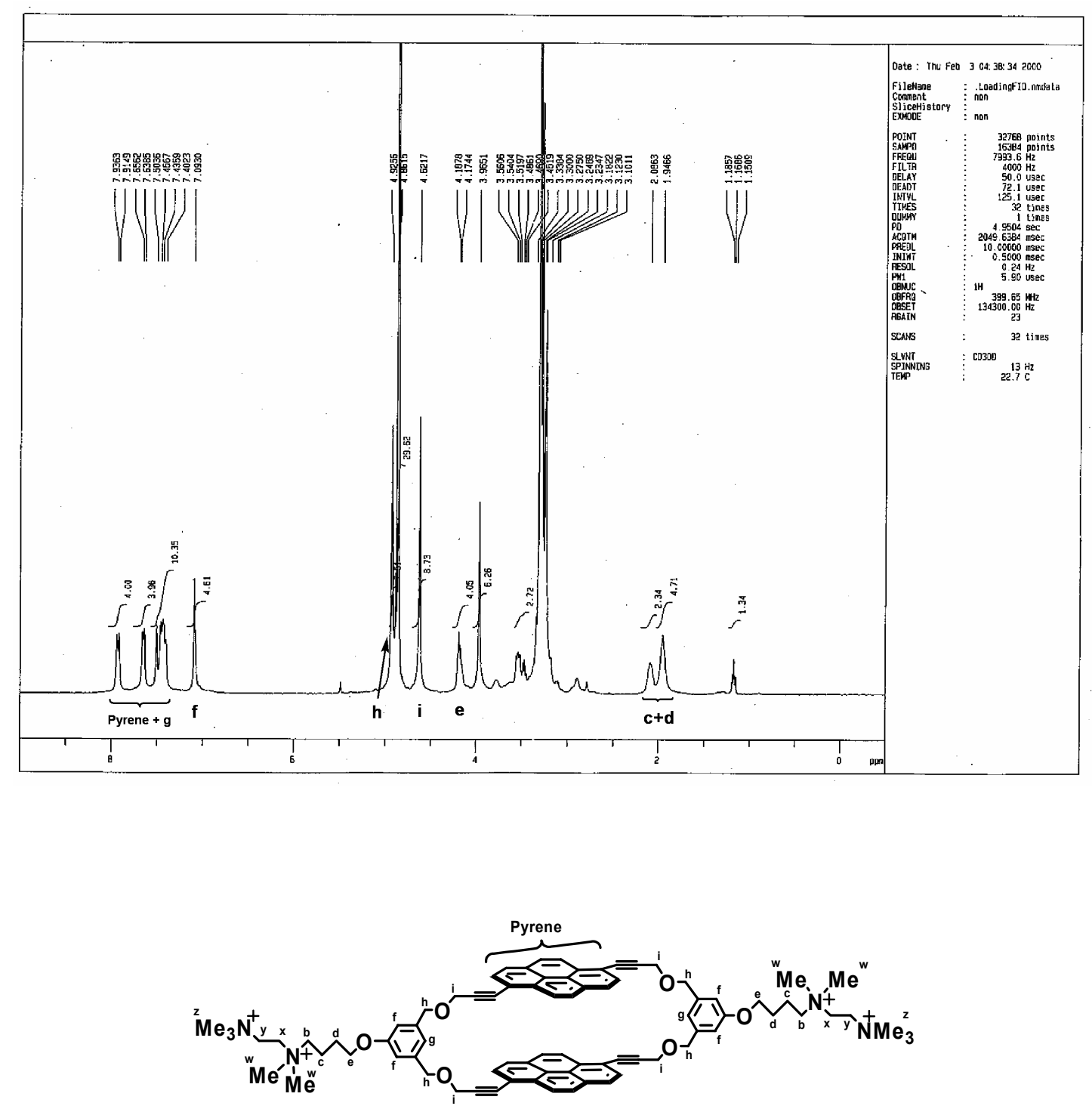

1d 
Diammonium Pyrenophane 1e
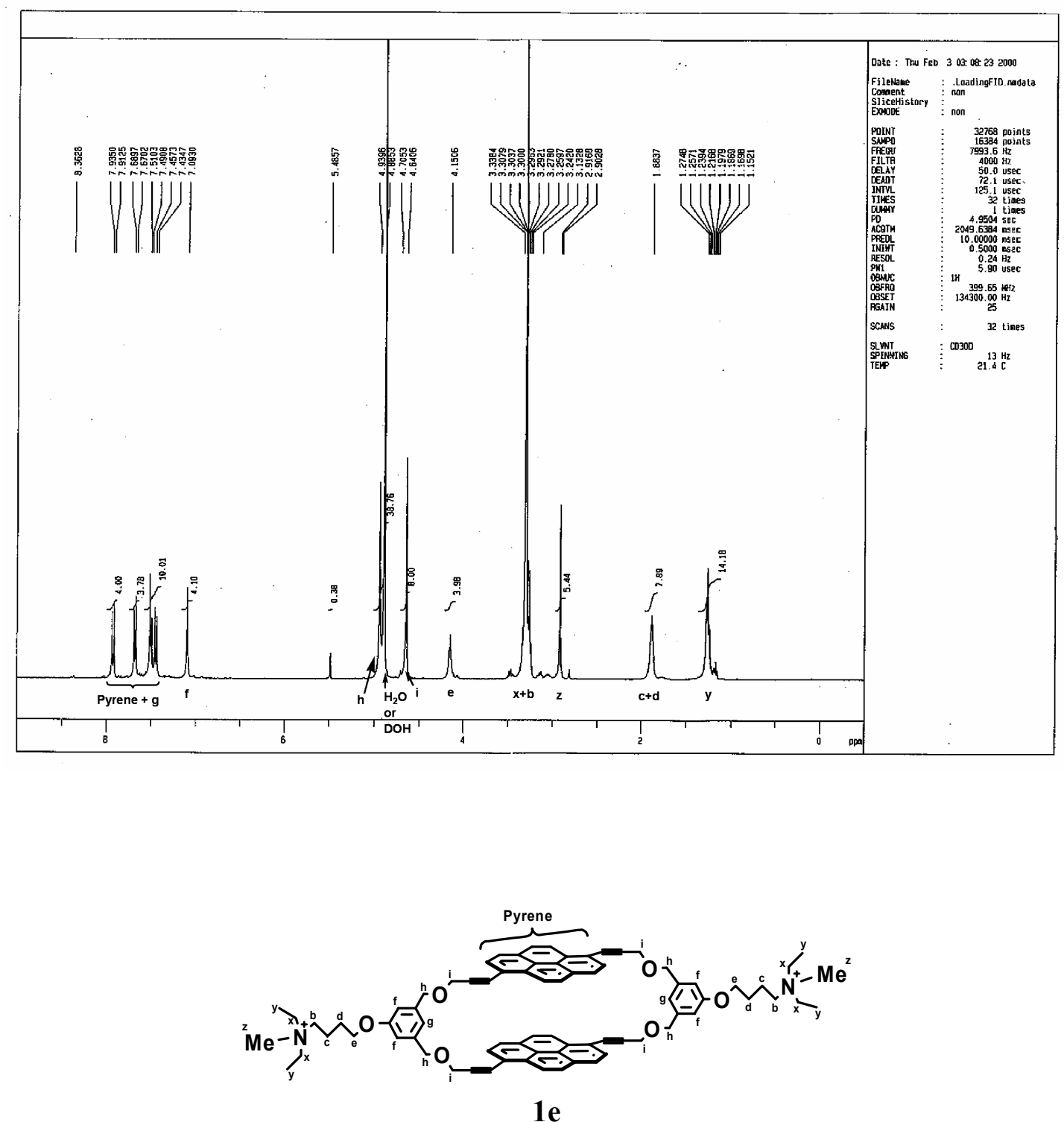
Diazoniacrown Pyrenophane 2a
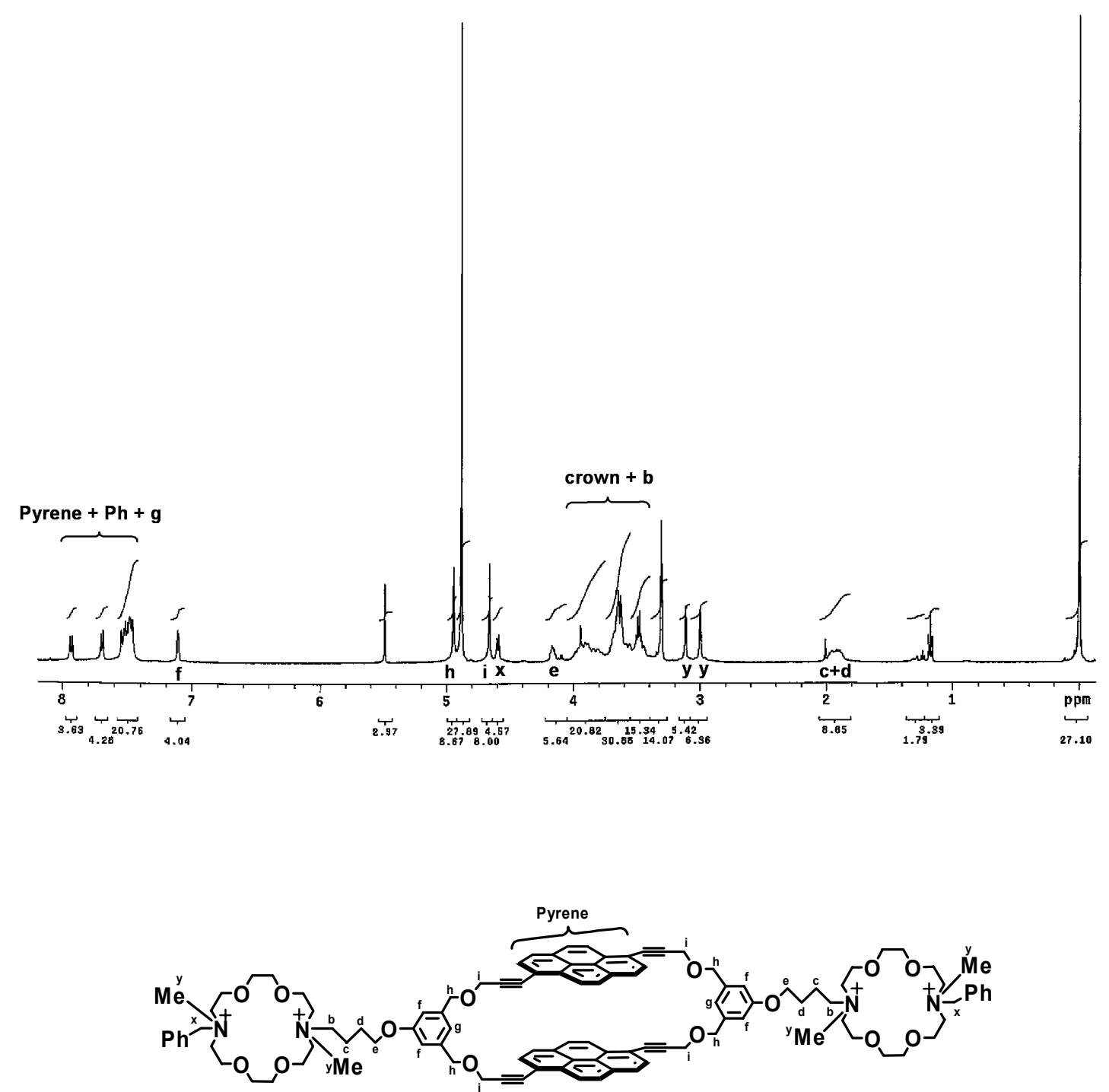

2a 
Poly(oxyethylene) Pyrenophane 3
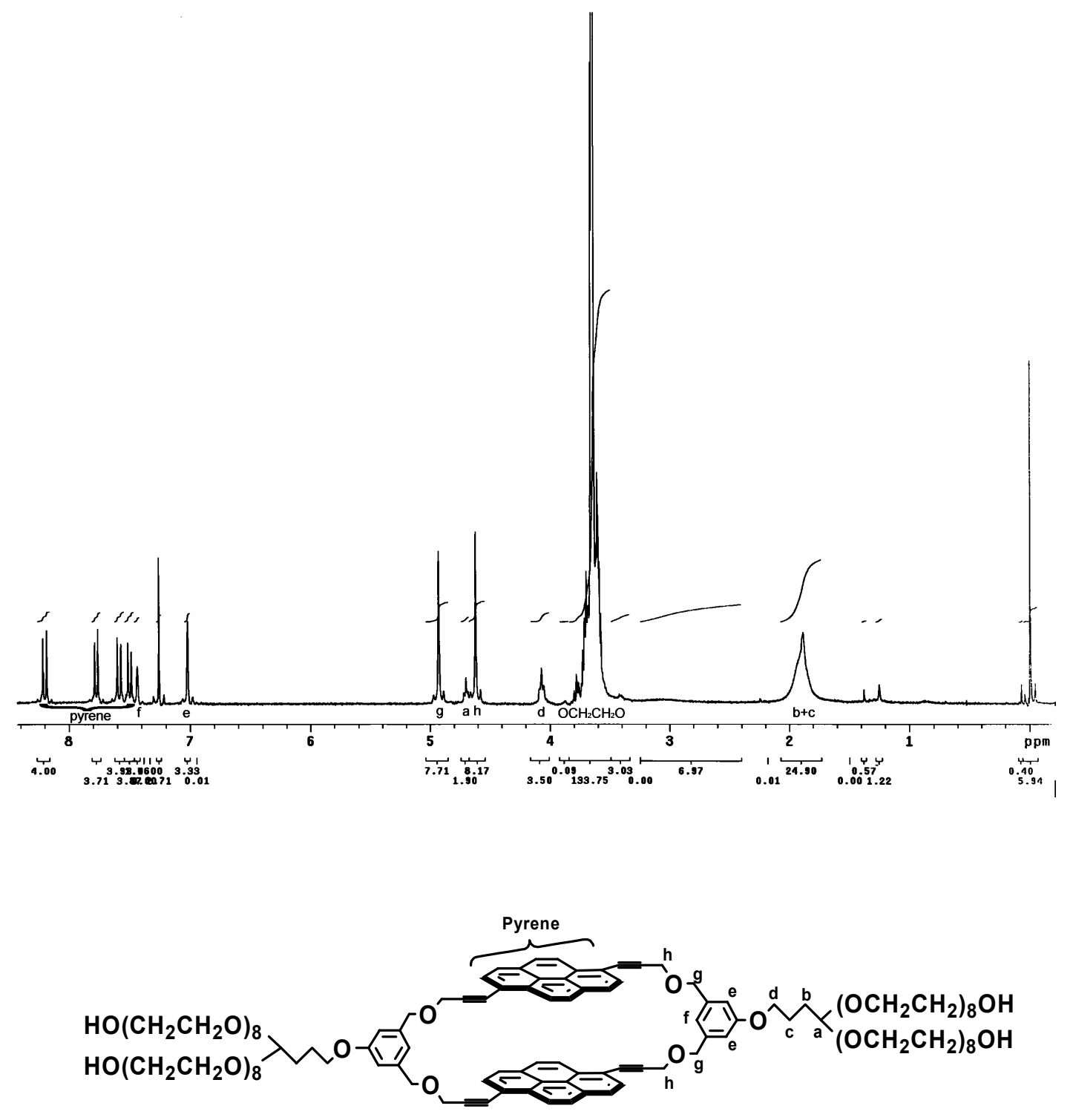

3 
1-[3-[Bis[3-(dimethylamino)propyl]amino]propyloxy]-3,5-bis(2-propynyloxymethyl)ben zene (5)
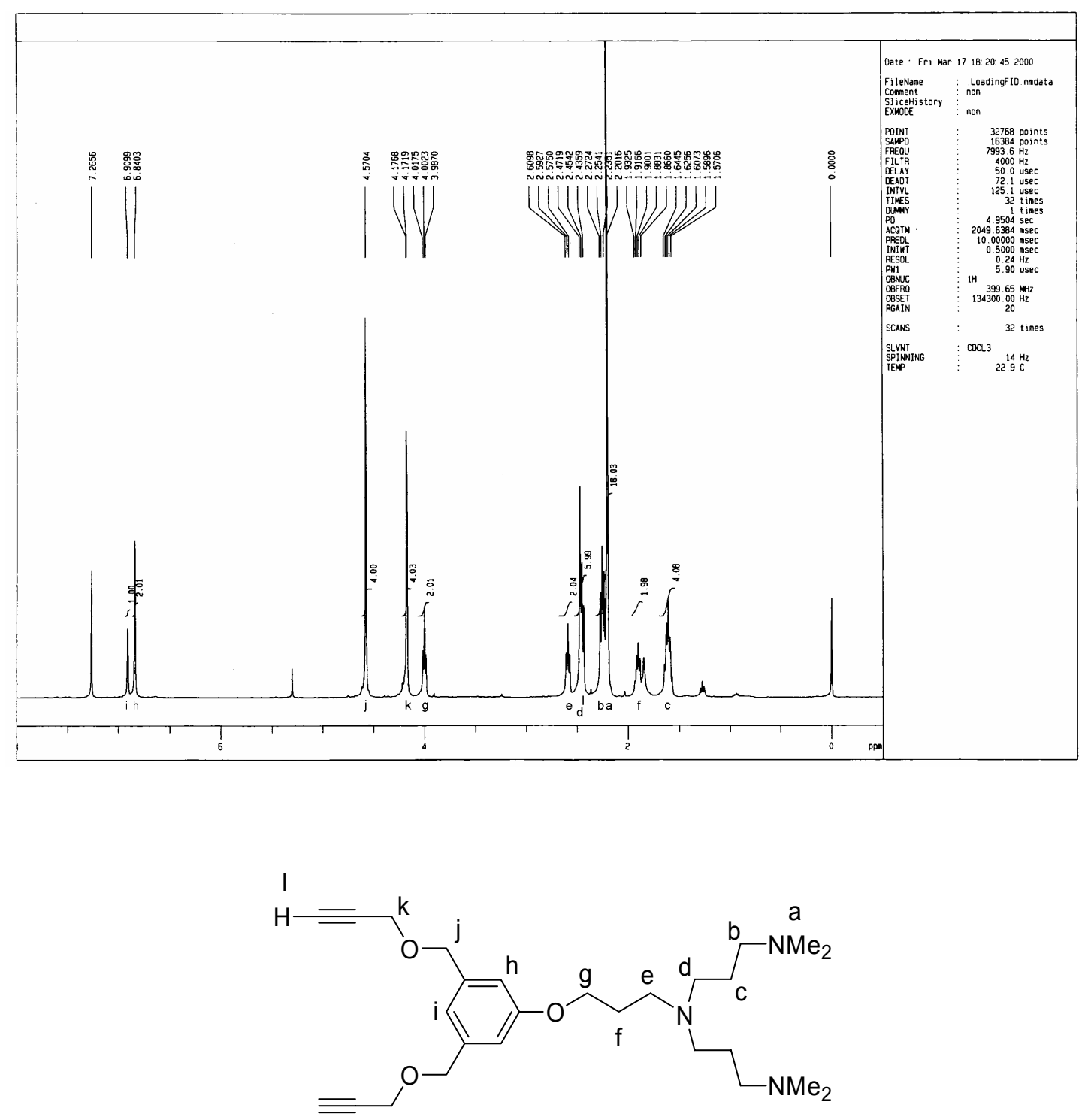

5 
1-[3-[Bis[3-(dimethylamino)propyl]amino]propyloxy]-3,5-bis[3-(tributylstannyl)-2-prop ynyloxymethyl]benzene (6):
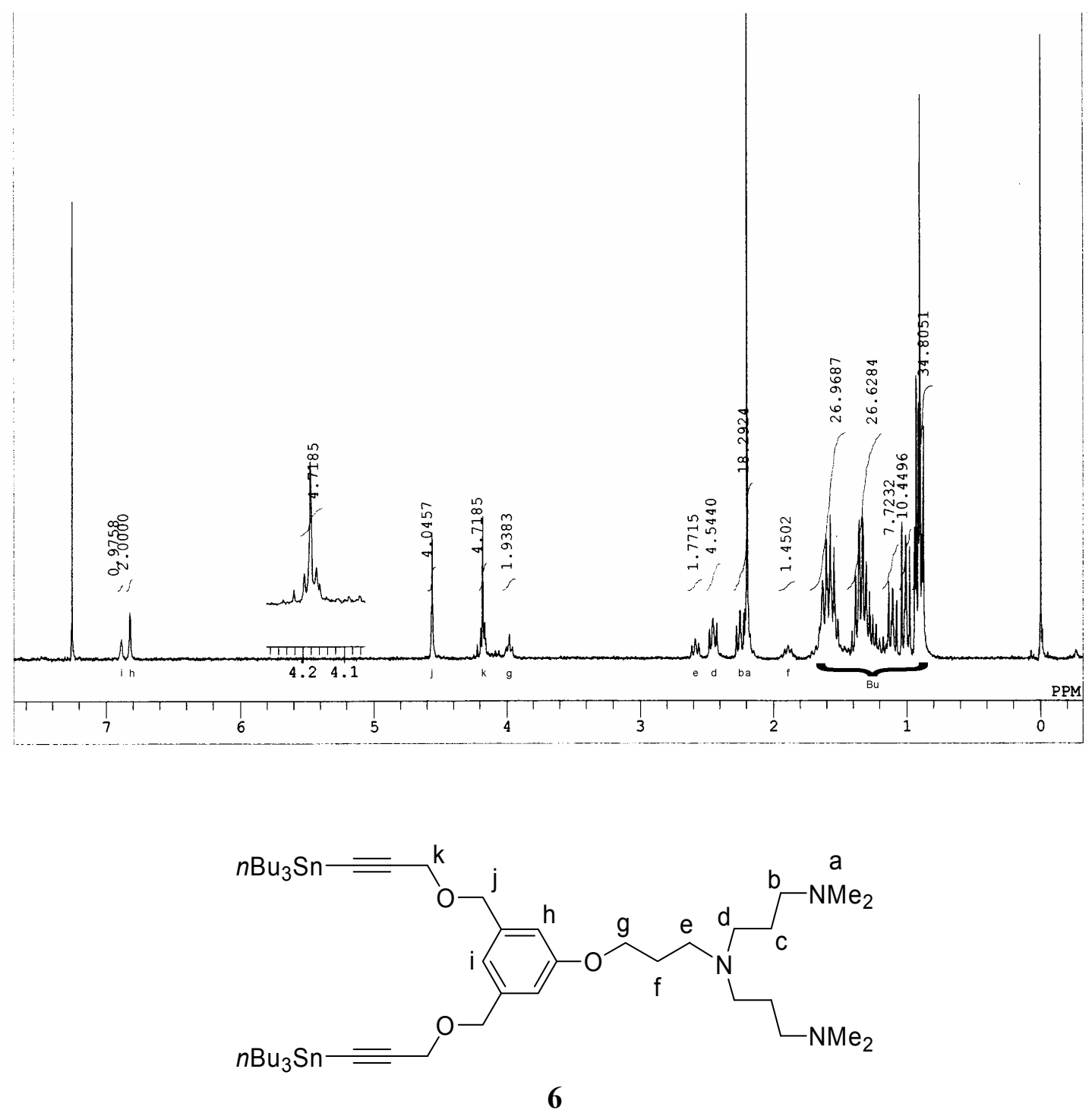
1-[3-[Bis[3-(dimethylamino)propyl]amino]propyloxy]-3,5-bis[3-(6-iodopyren-1-yl)-2-pr opynyloxymethyl]benzene (7)
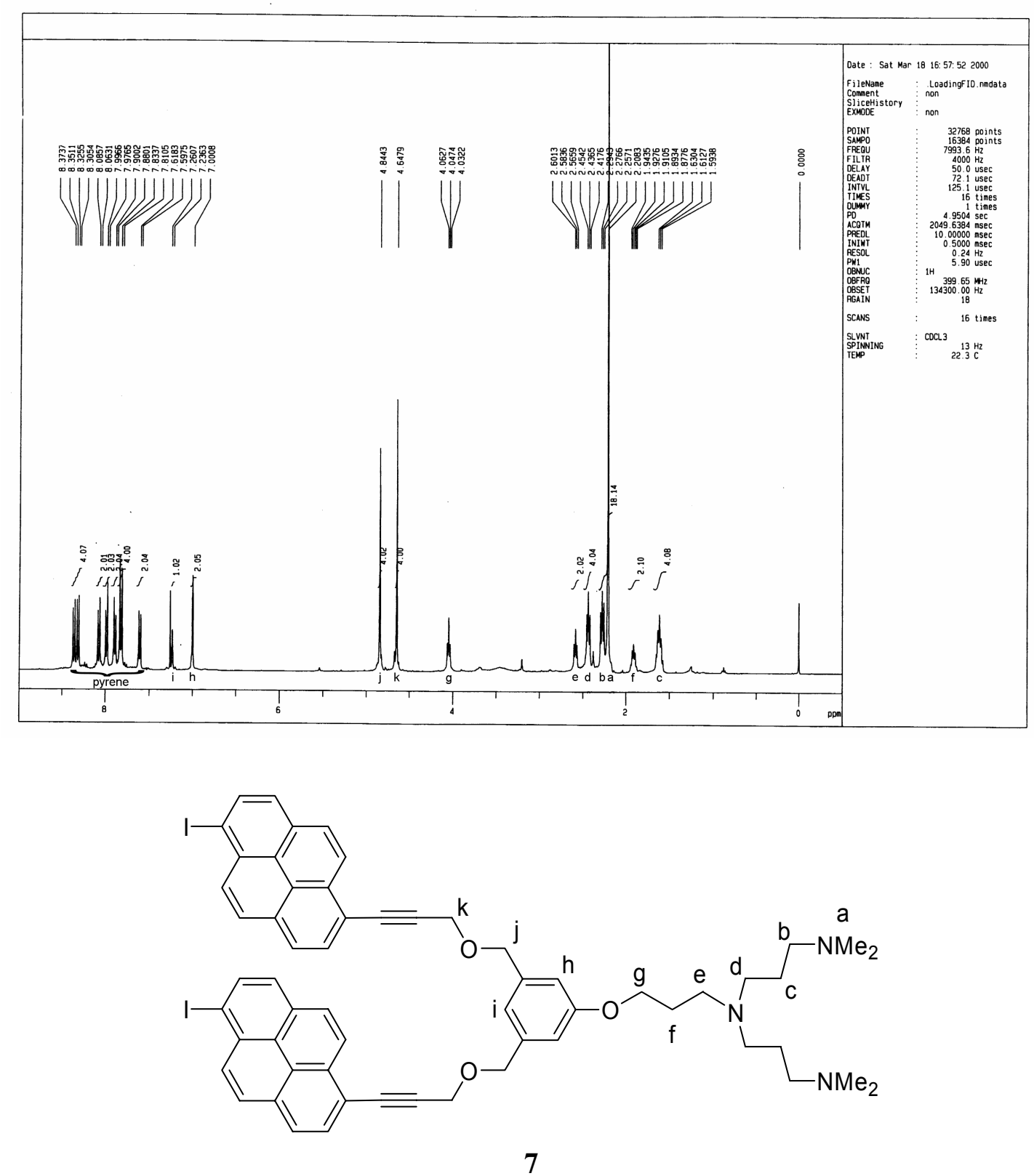
Hexaamino Pyrenophane 8
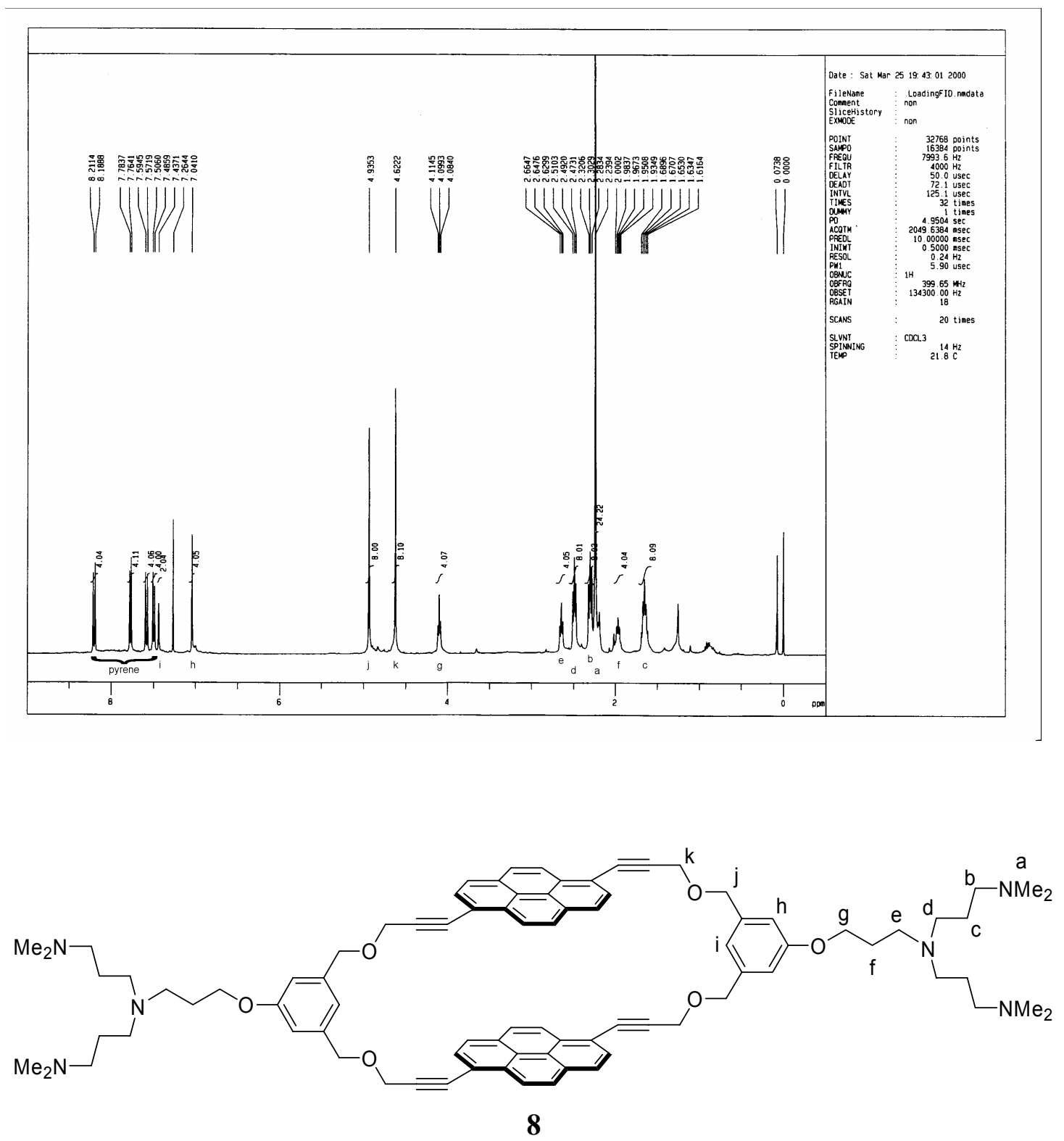
1-(Triisopropylsiloxy)-3,5-bis(2-propynyloxymethyl)benzene (9)
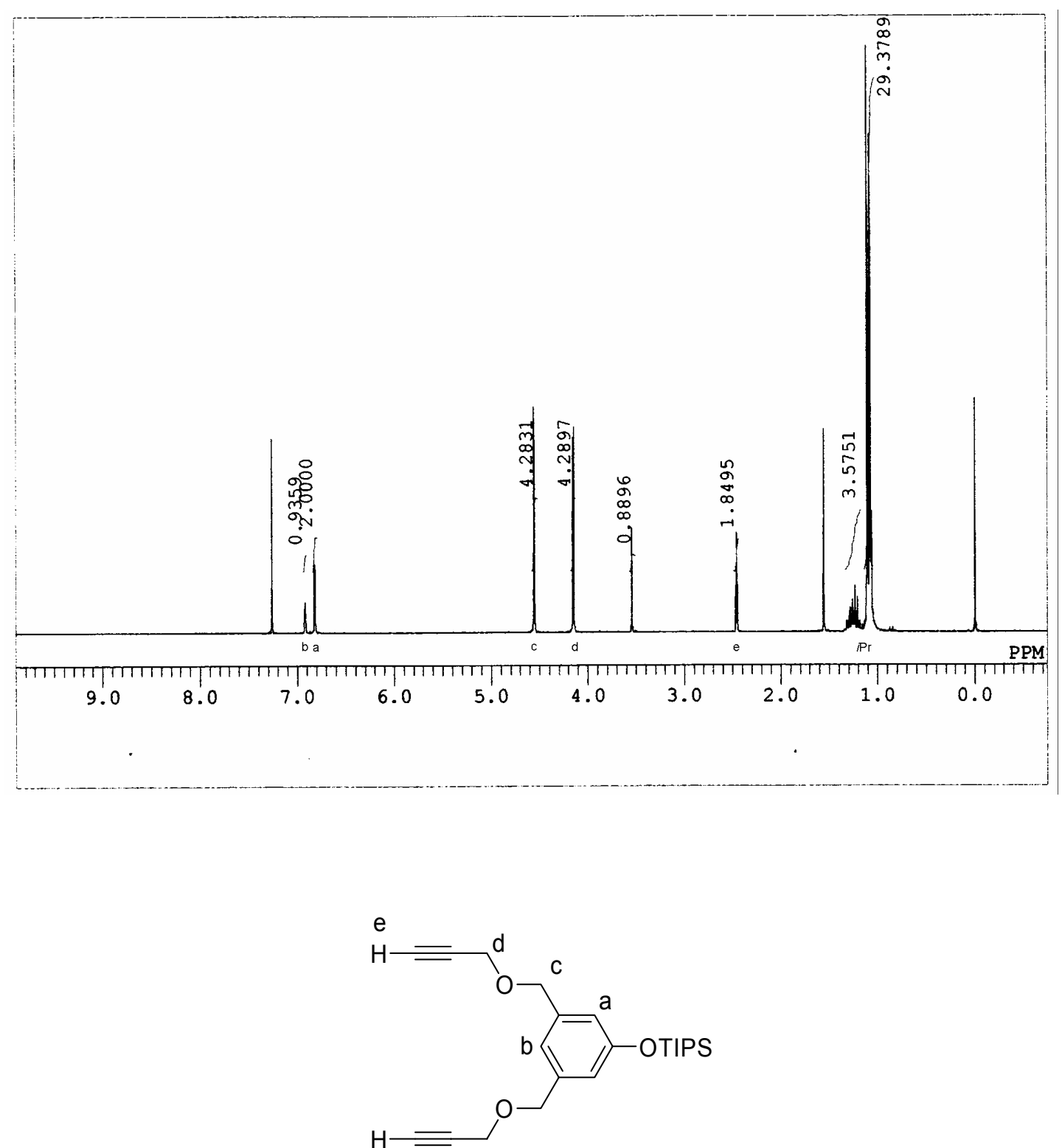
1-(Triisopropylsiloxy)-3,5-bis(3-tributylstannyl-2-propynyloxymethyl)benzene (10)
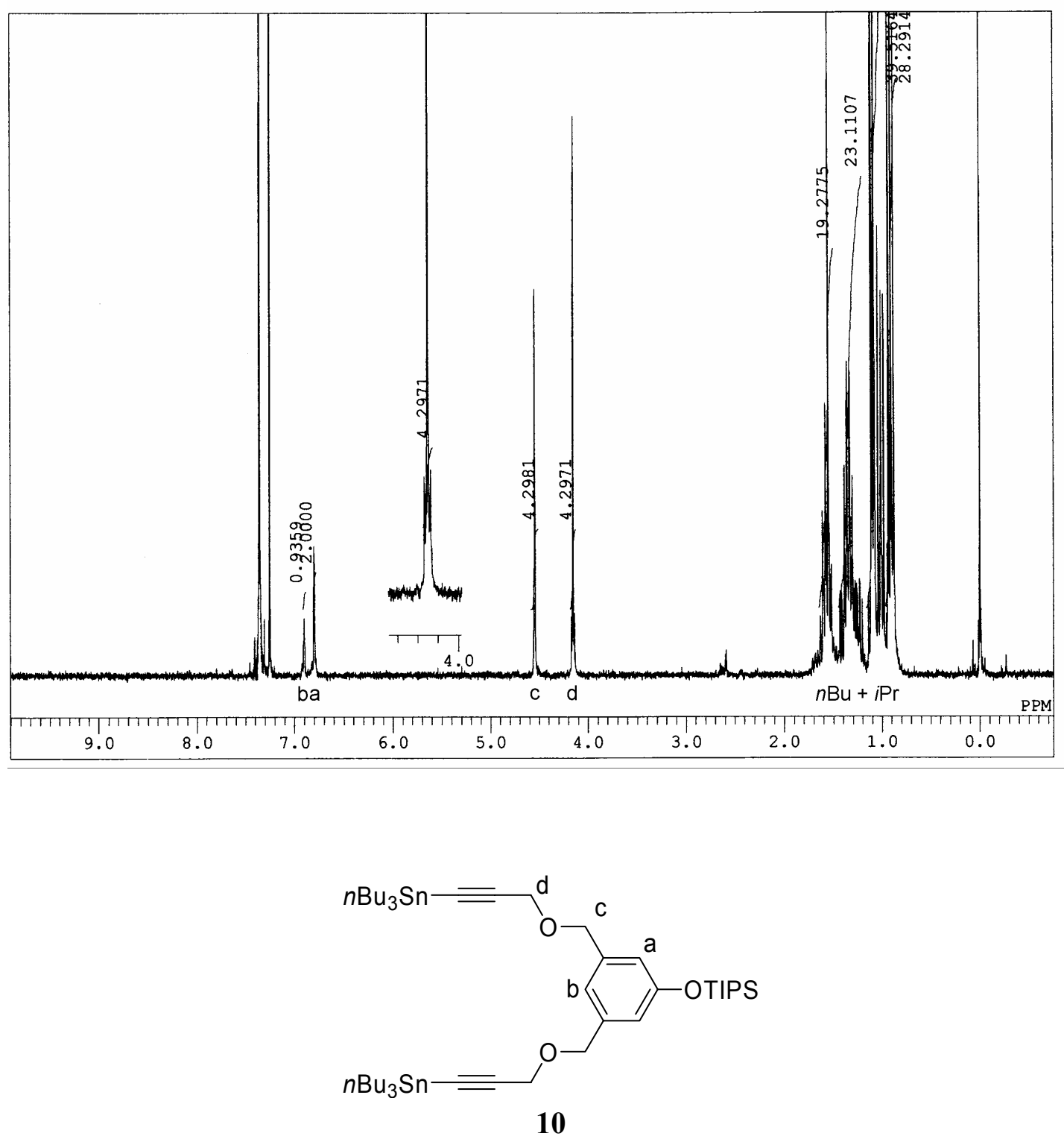

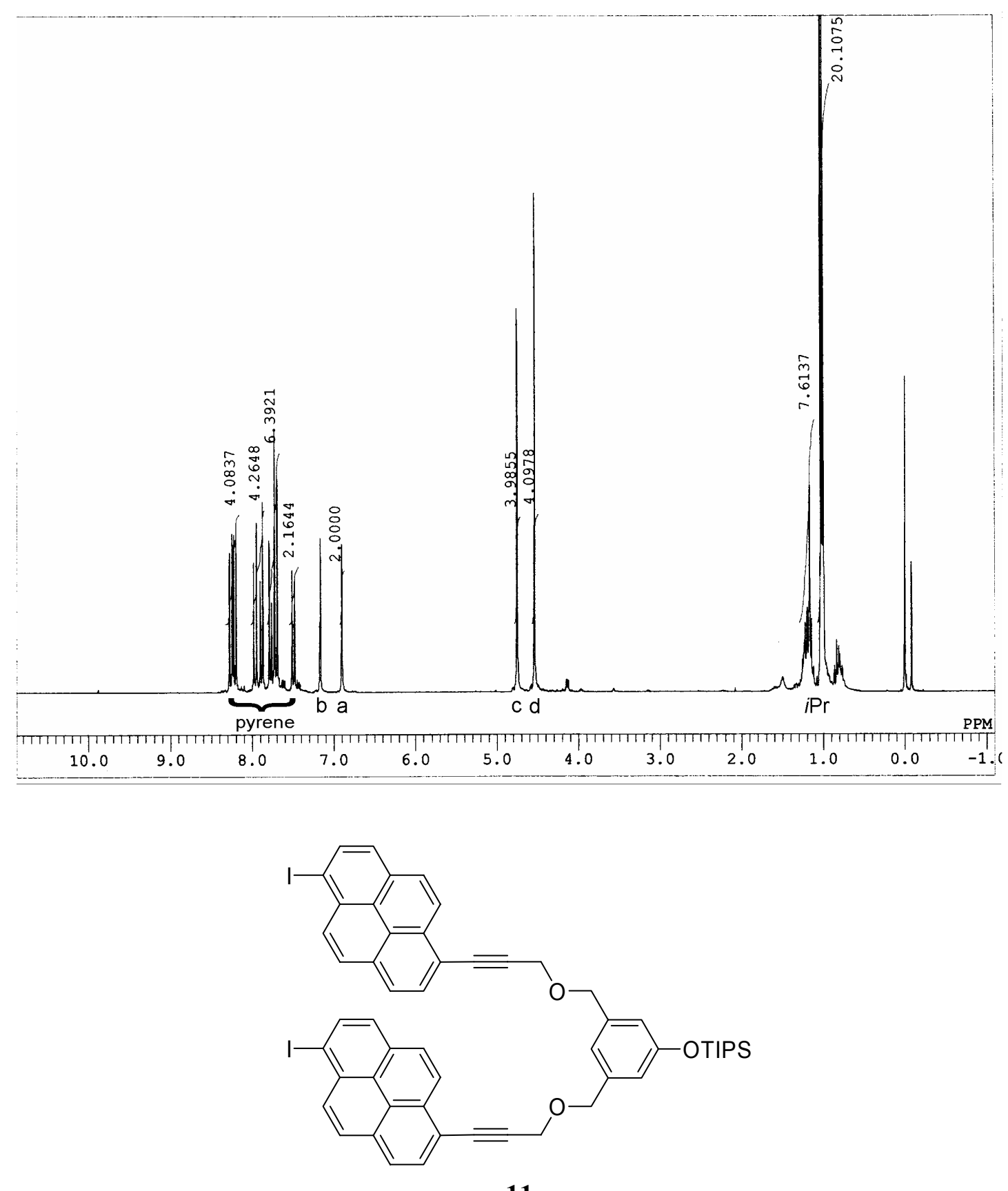

11 
Triisopropylsilyl Pyrenophane $\mathbf{1 2}$
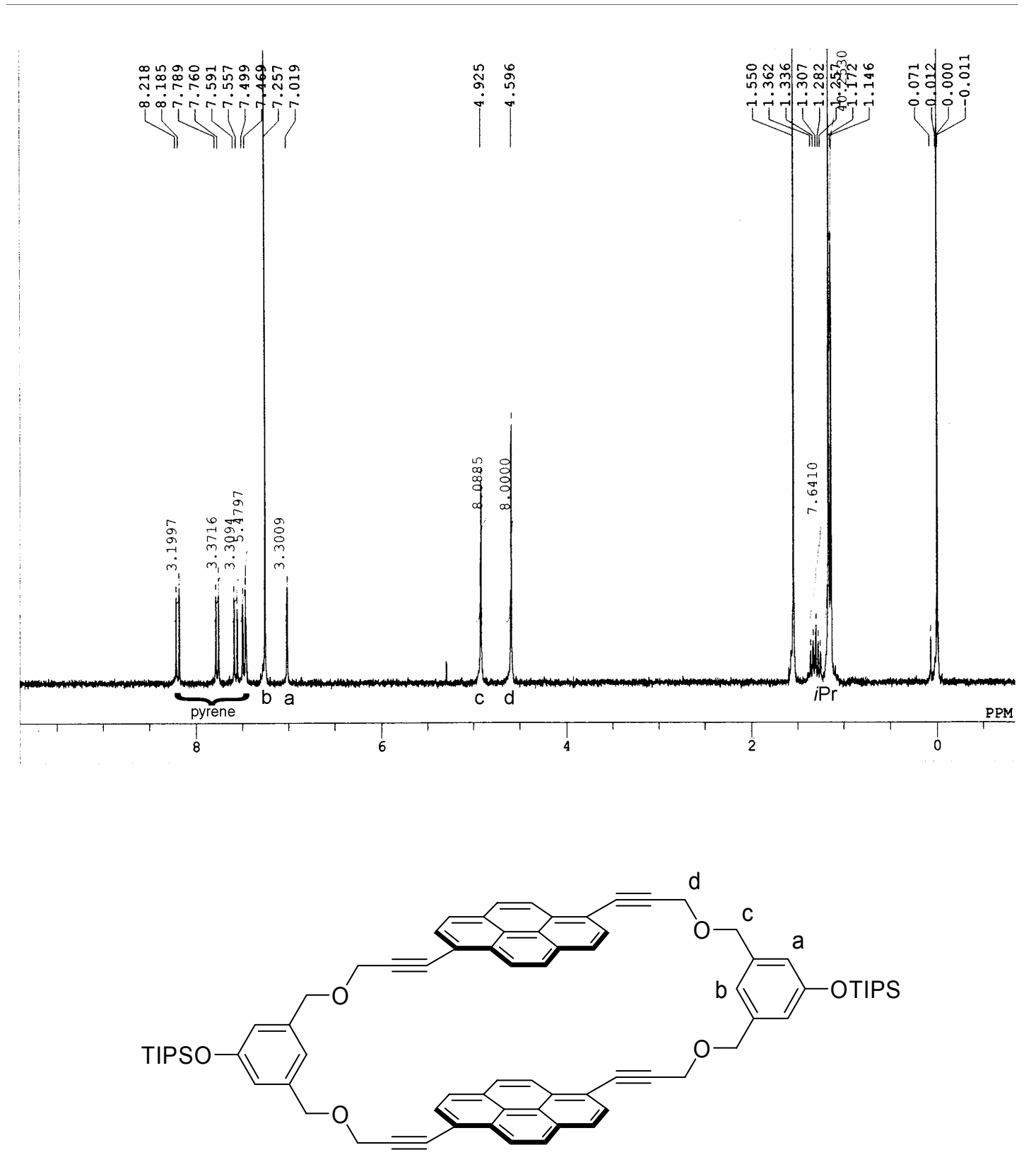

12 
Phenolic Pyrenophane 13
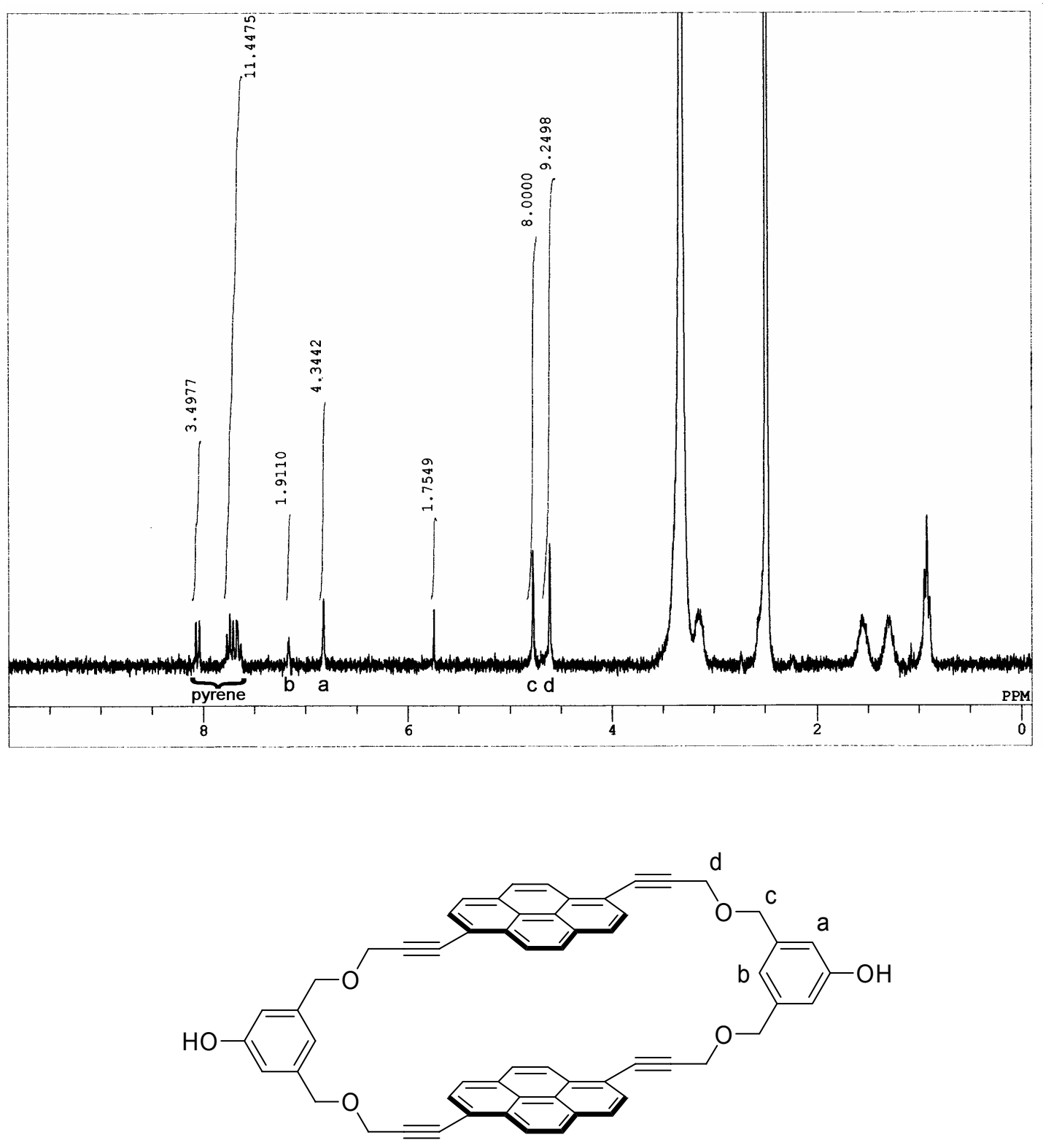

13 
1-(4,4-Dimethoxybutyloxy)-3,5-bis(hydroxymethyl)benzene
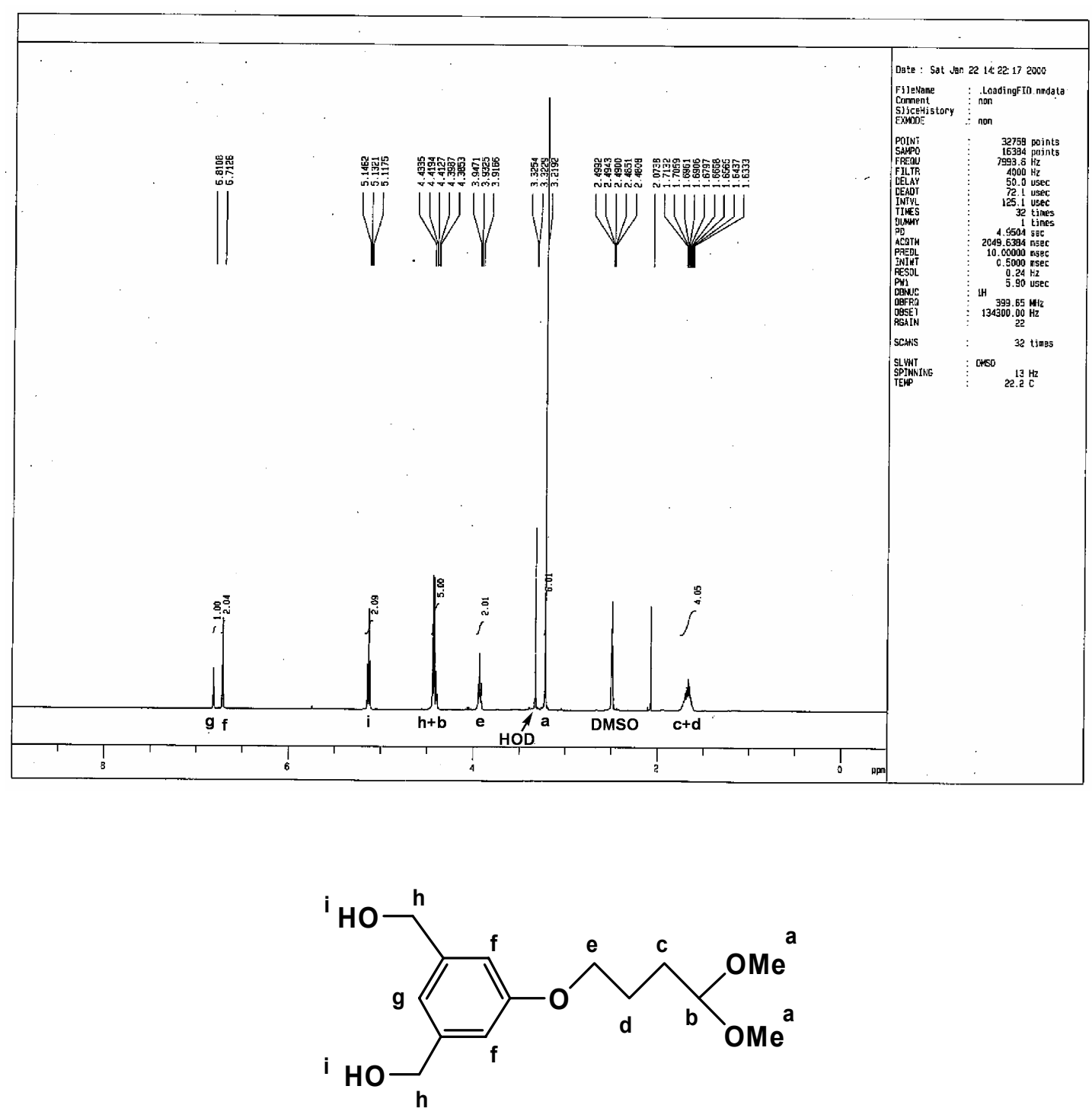
1-(4,4-Dimethoxybutyloxy)-3,5-bis(2-propynyloxymethyl)benzene (14)
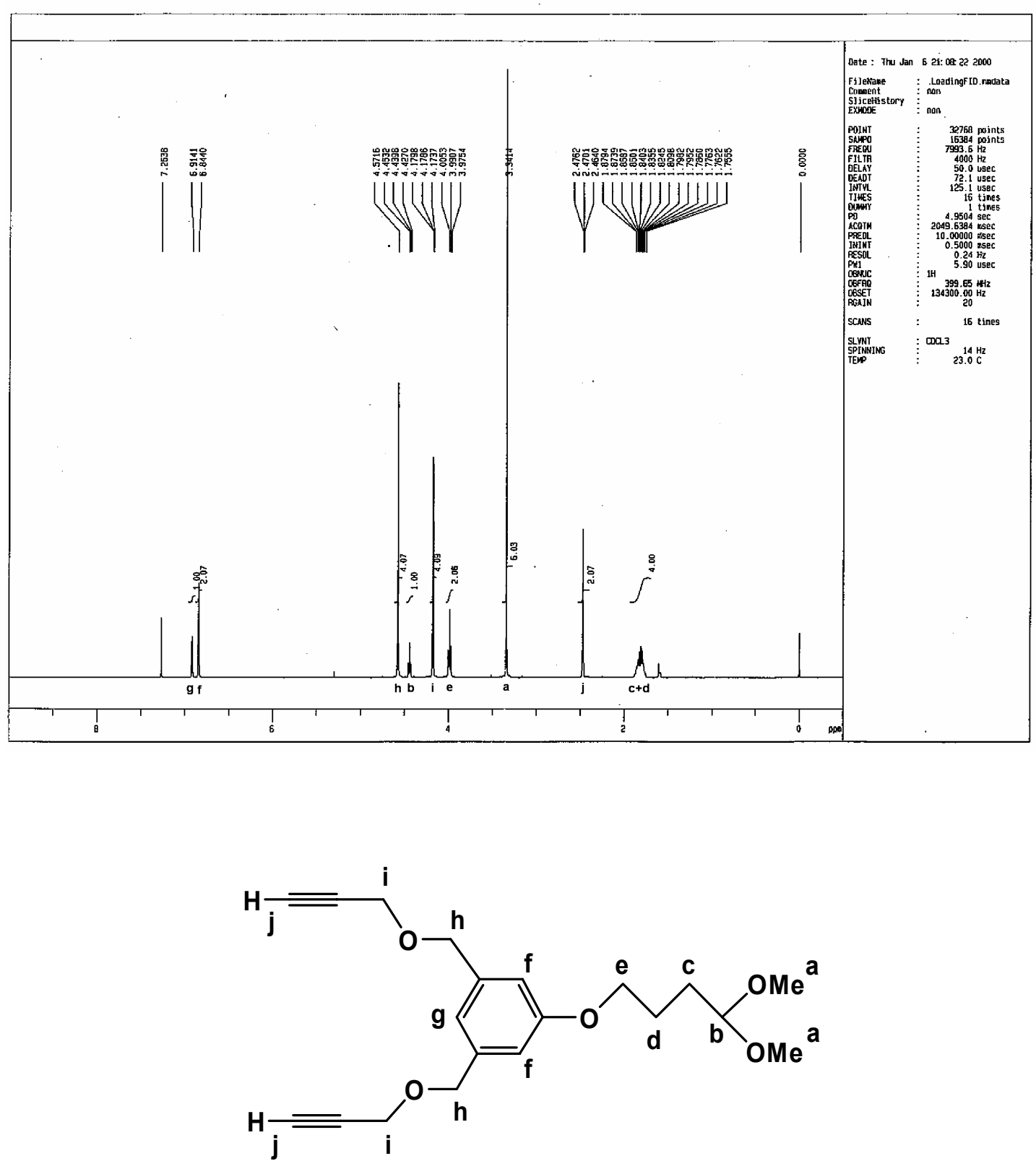

14 

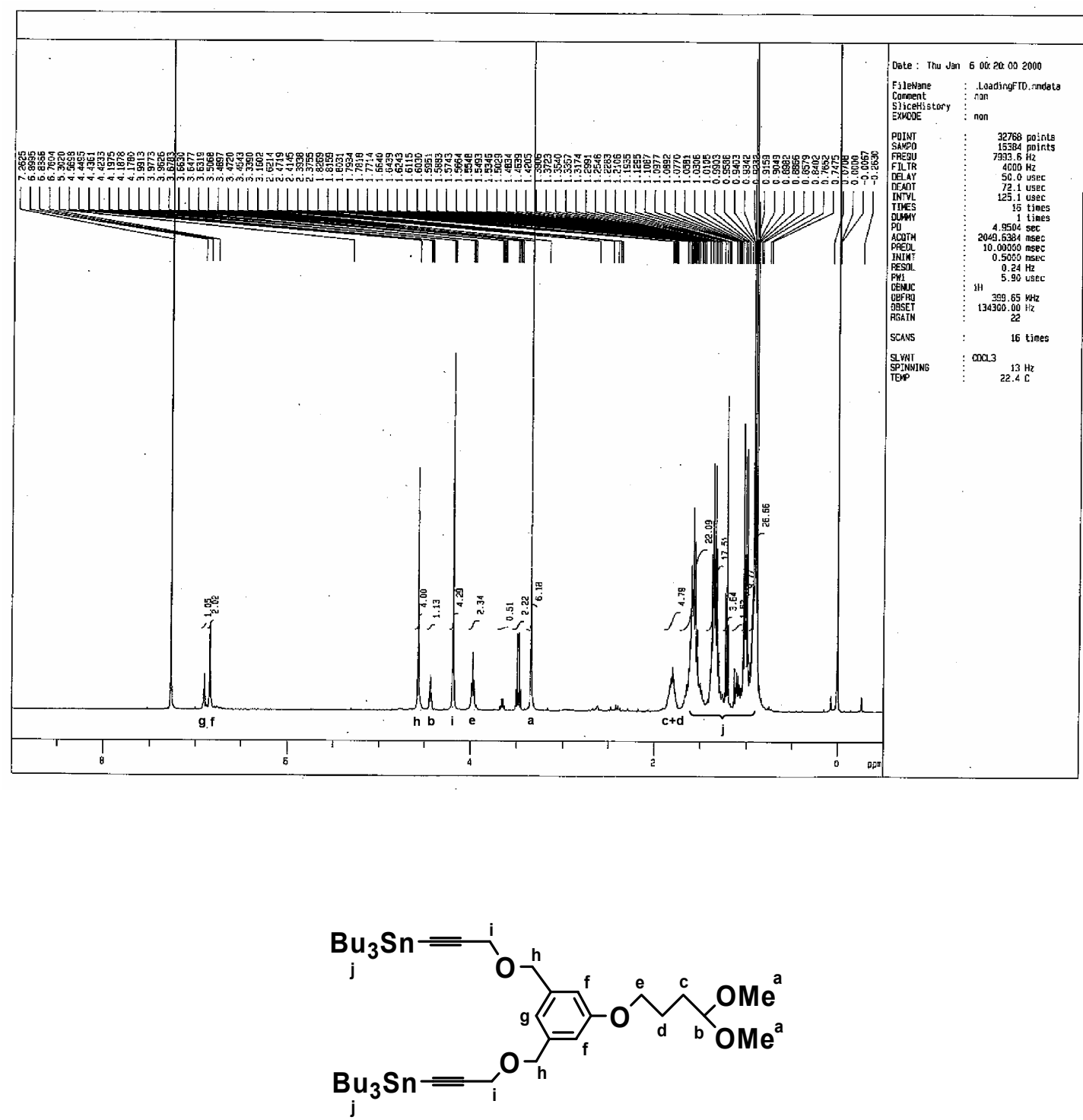

15 
1-(4,4-Dimethoxybutyloxy)-3,5-bis[3-(6-iodopyren-1-yl)-2-propynyloxymethyl]benzene (16)
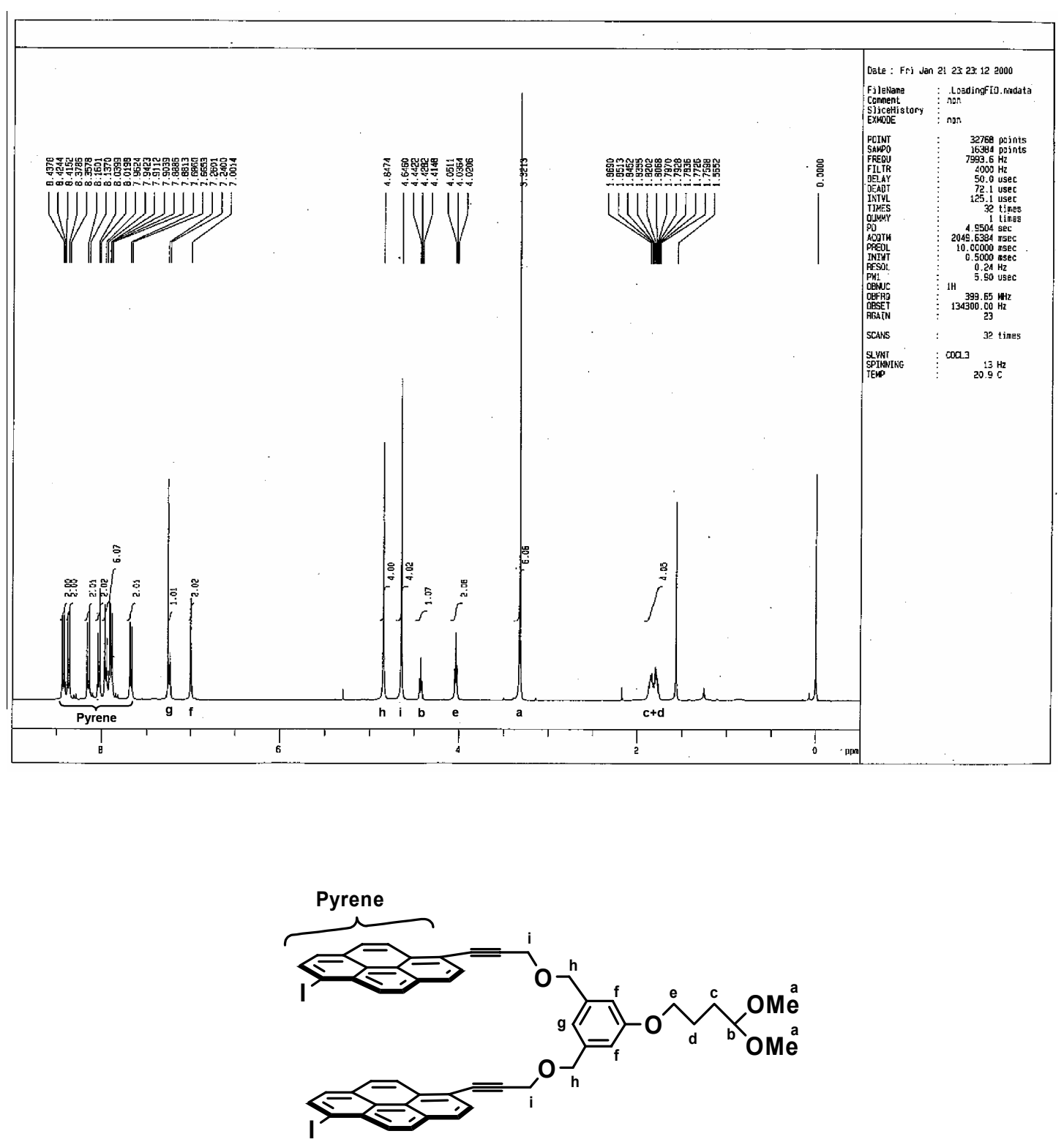

16 
Acetalic Pyrenophane 17
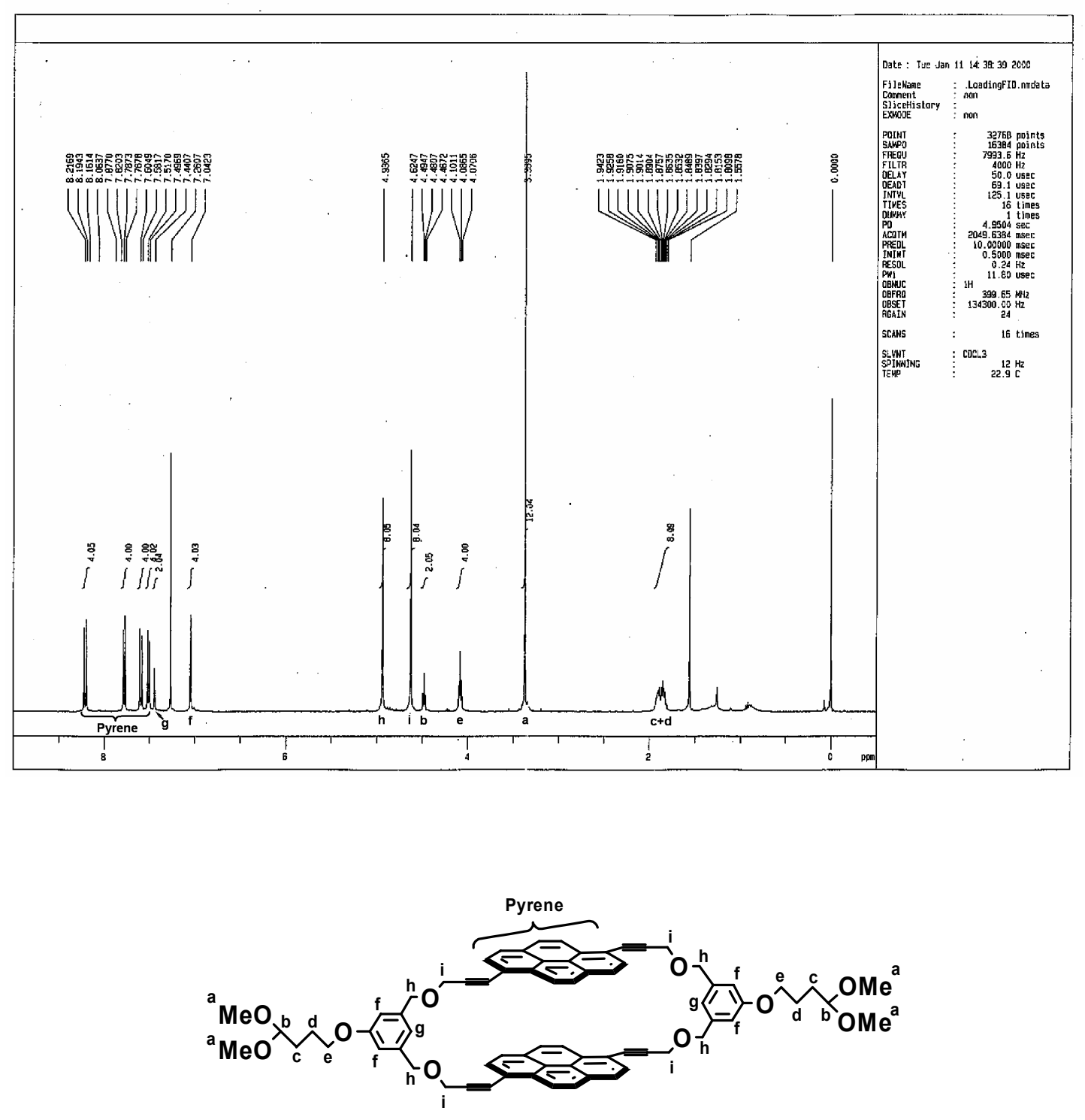

17 
Aldehydic Pyrenophane 18
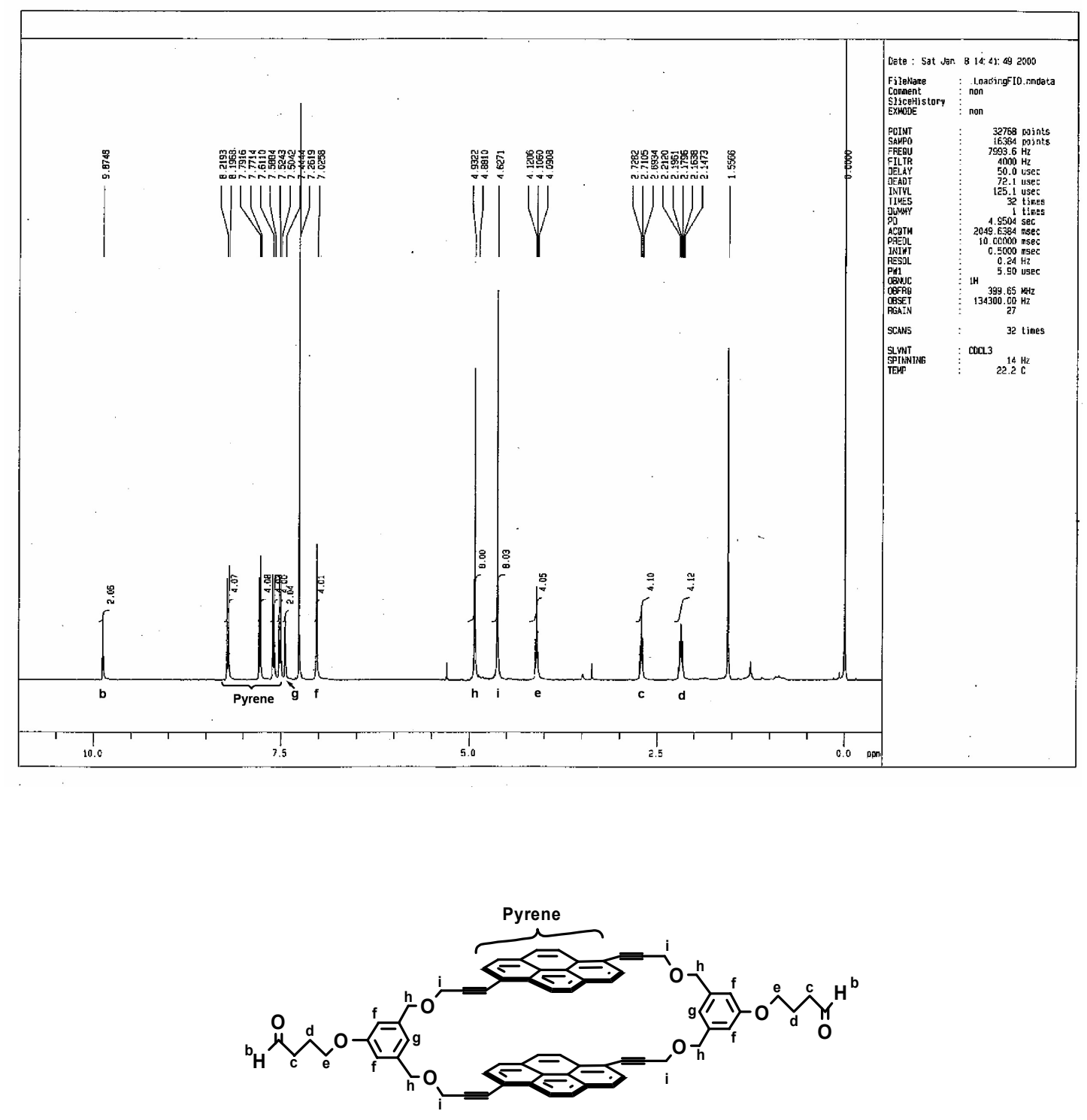

18 
Hexaamino Pyrenophane 19b
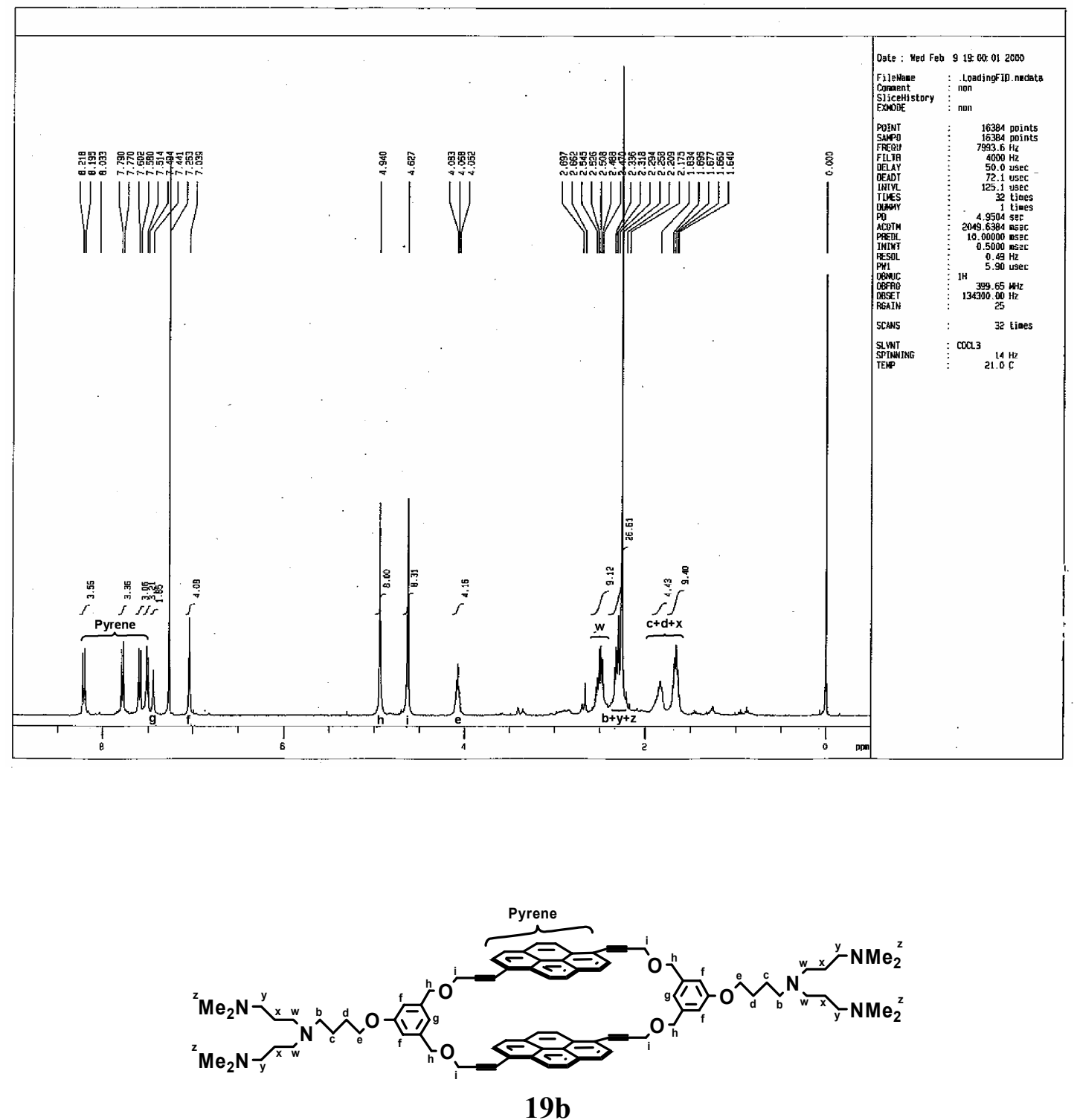
Tetraamino Pyrenophane 19d
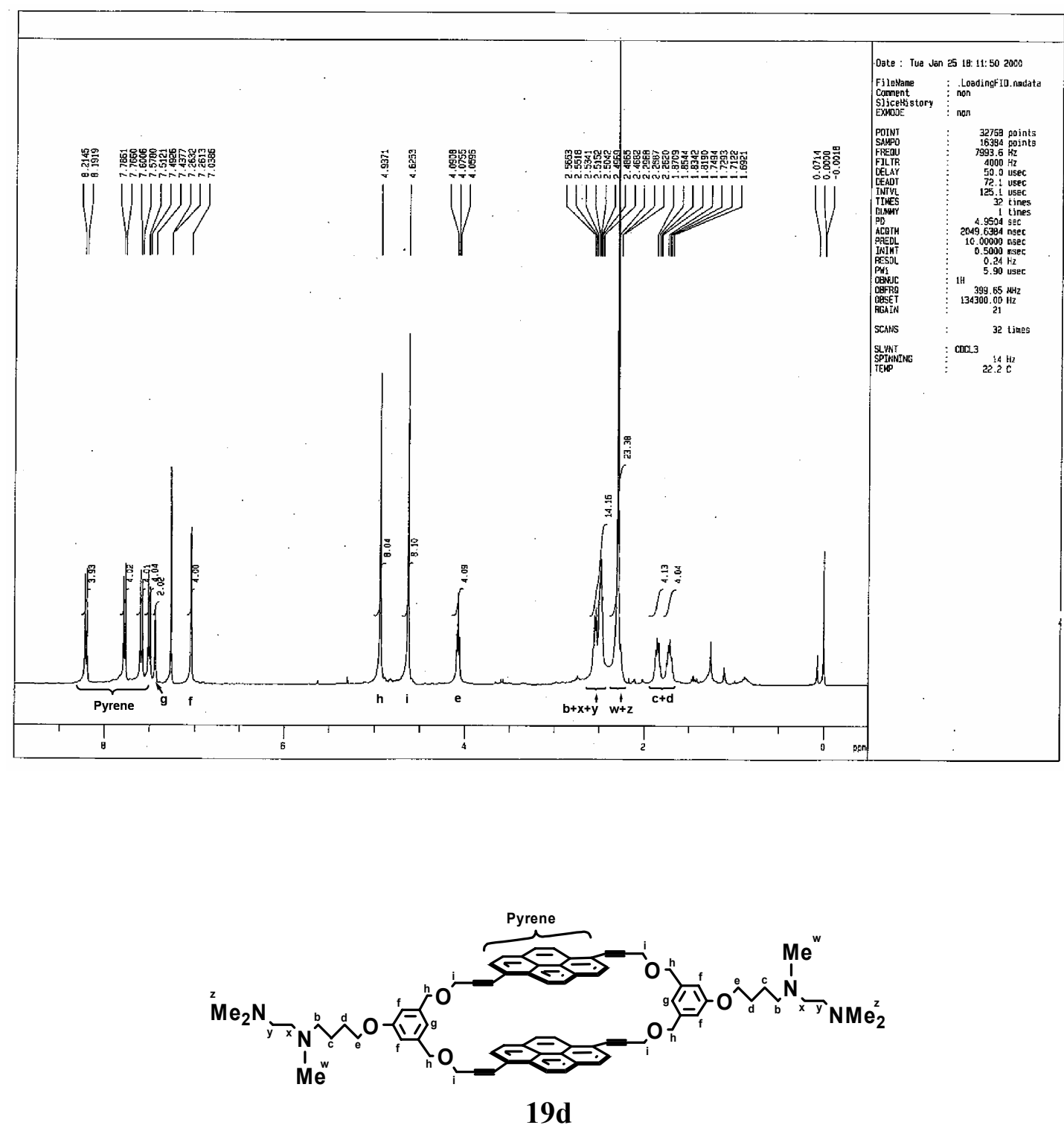
Diamino Pyrenophane 19e
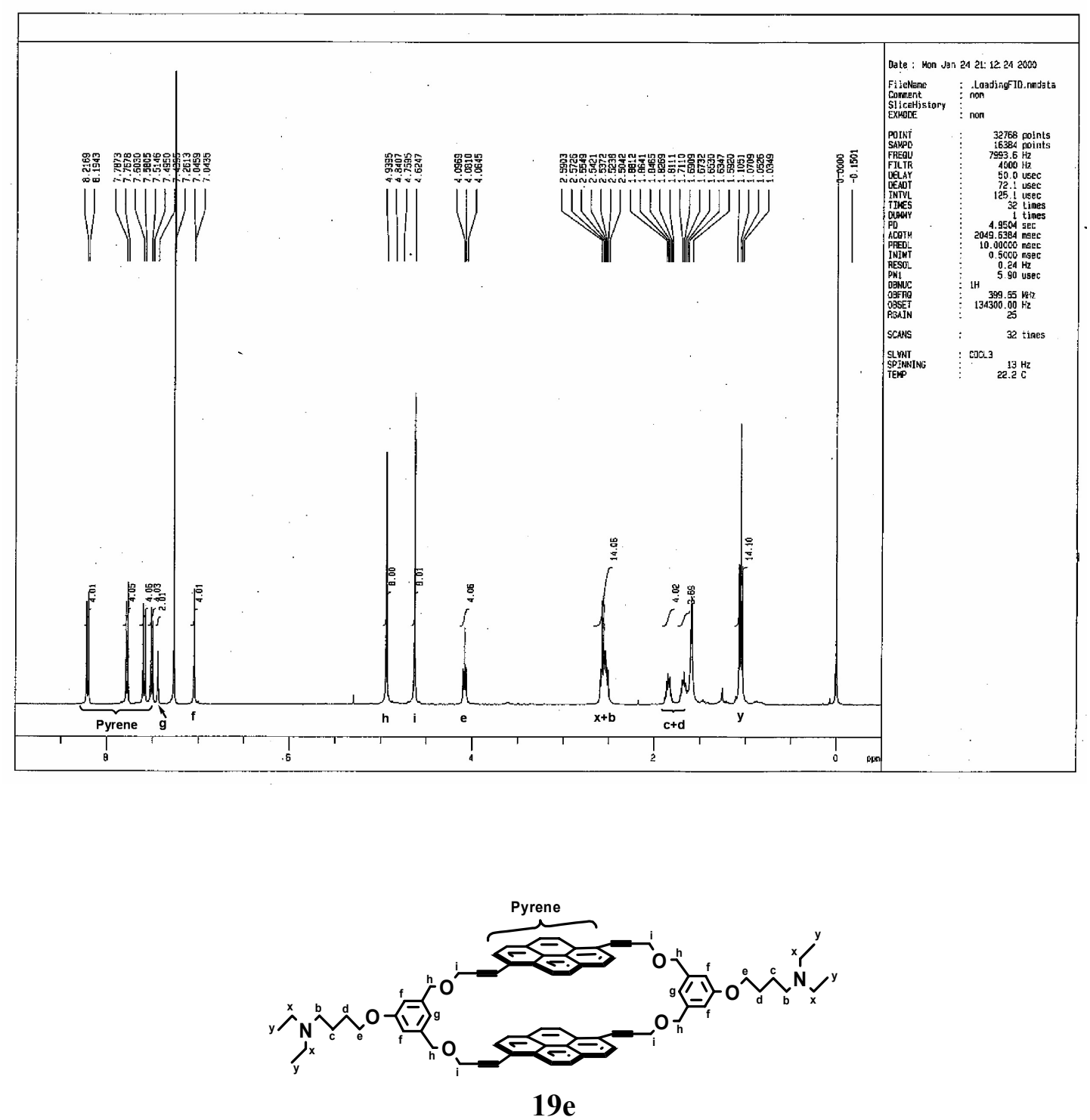
Diazacrown Pyrenophane 20a
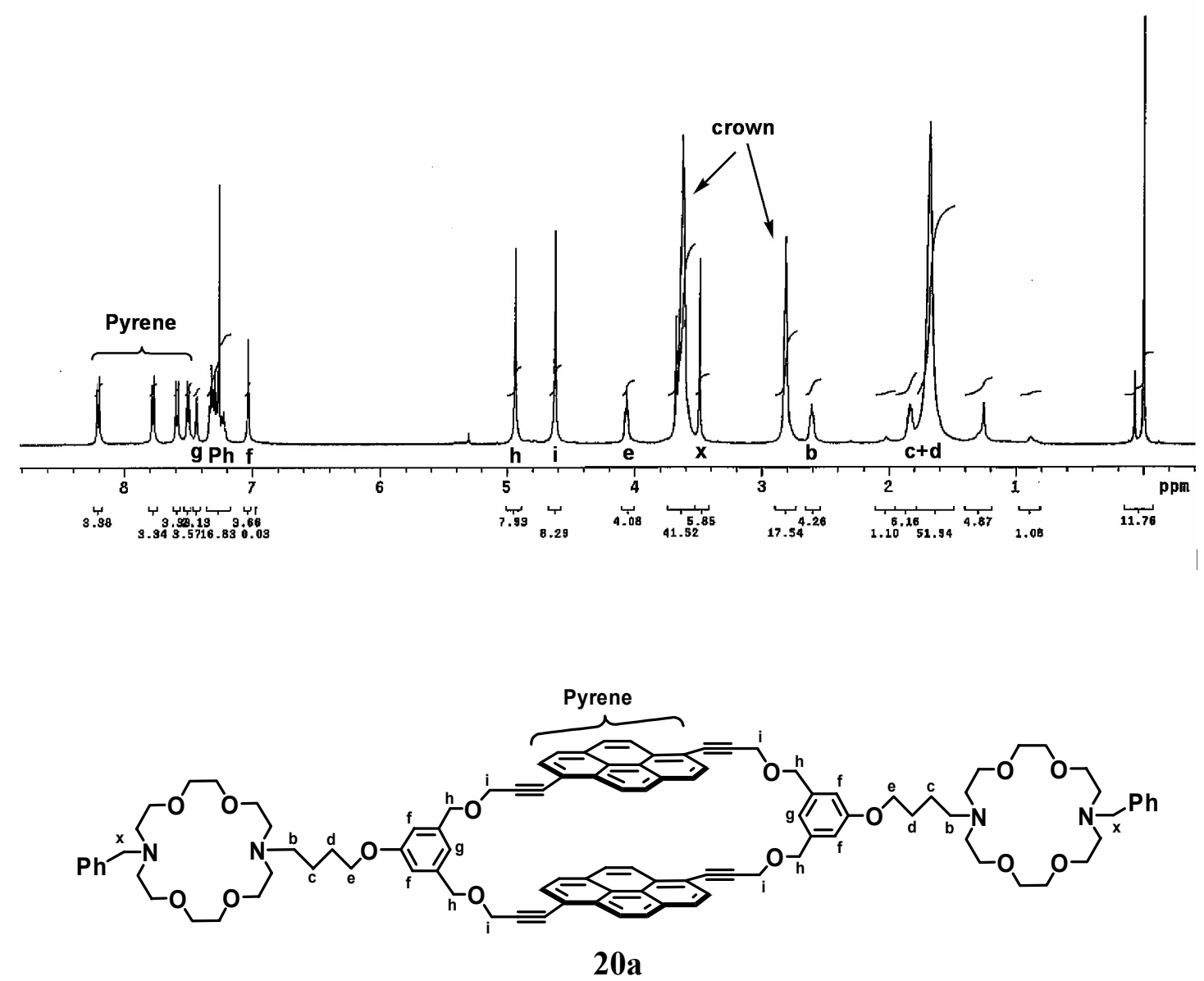
Azacrown Pyrenophane 20b
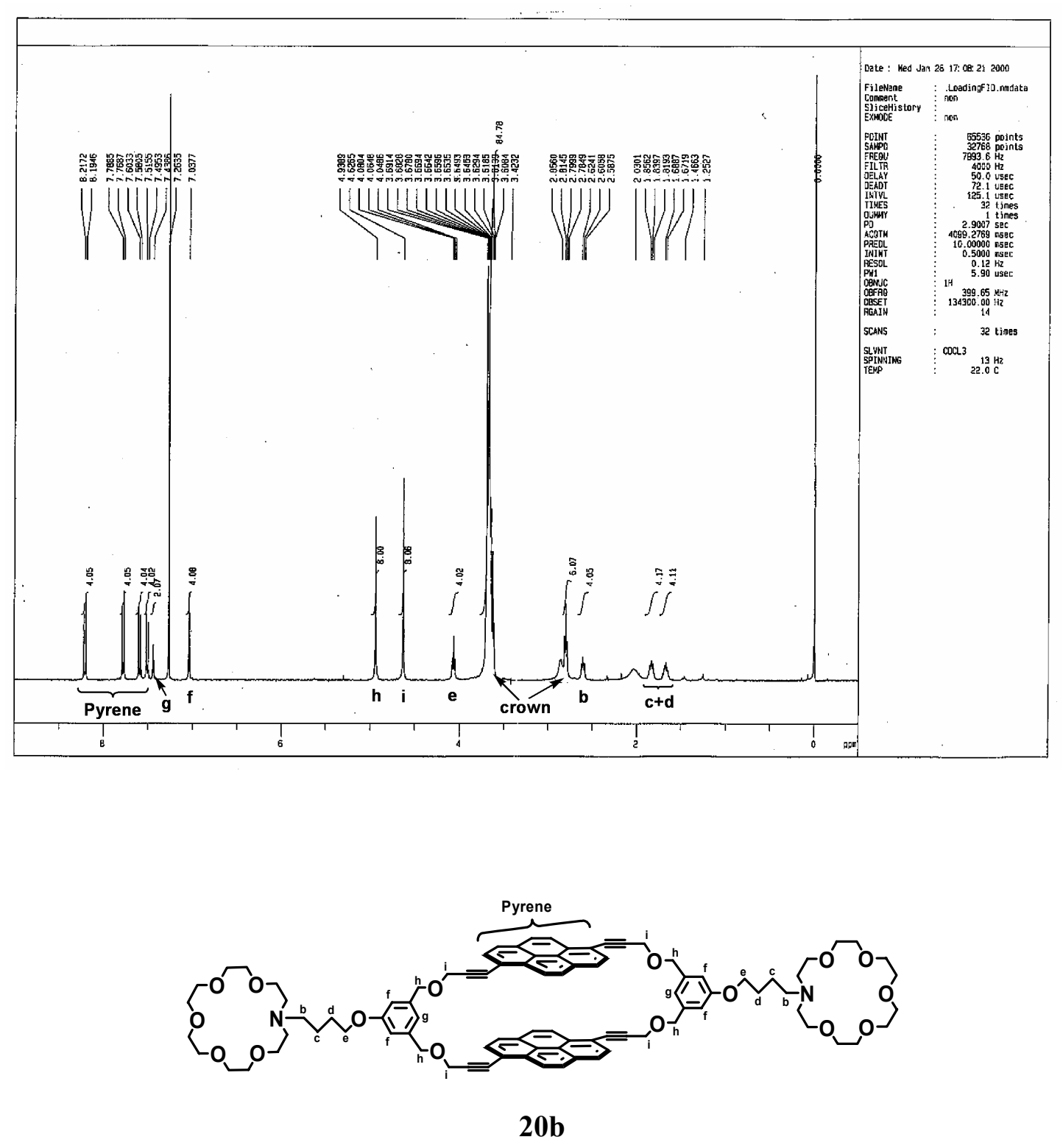